\title{
DRAWING GRAPHS ON FEW LINES AND FEW PLANES*
}

\author{
Steven Chaplick, ${ }^{\dagger \ddagger}$ Krzysztof Fleszar, ${ }^{\S}$ Fabian Lipp, ${ }^{\dagger}$ Alexander Ravsky, $\|$ Oleg Verbitsky, ${ }^{* *}$ \\ and Alexander Wolfft†
}

ABstract. We investigate the problem of drawing graphs in $2 \mathrm{D}$ and $3 \mathrm{D}$ such that their edges (or only their vertices) can be covered by few lines or planes. We insist on straightline edges and crossing-free drawings. This problem has many relations to other challenging graph-drawing problems such as small-area or small-volume drawings, layered or track drawings, and drawing graphs with low visual complexity. While some facts about our problem are implicit in previous work, this is the first treatment of the problem in its full generality. Our contribution is as follows.

- We show lower and upper bounds for the numbers of lines and planes needed for covering drawings of graphs in certain graph classes. In some cases our bounds are asymptotically tight; in some cases we are able to determine exact values.

- We relate our parameters to standard combinatorial characteristics of graphs (such as the chromatic number, treewidth, or arboricity) and to parameters that have been studied in graph drawing (such as the track number or the number of segments appearing in a drawing).

- We pay special attention to planar graphs. For example, we show that there are planar graphs that can be drawn in 3-space on asymptotically fewer lines than in the plane.

\section{Introduction}

It is well known that any graph admits a crossing-free straight-line drawing in 3-space. Suppose that we are allowed to draw edges only on a limited number of planes. How many

${ }^{*}$ A preliminary version of this paper appeared in the Proceedings of the 24th International Symposium on Graph Drawing and Network Visualization (GD 2016) [17].

${ }^{\dagger}$ Lehrstuhl für Informatik I, Julius-Maximilians-Universität Würzburg, Germany, www.informatik.uniwuerzburg.de/en/algo/staff, firstname.lastname@uni-wuerzburg.de

${ }_{\ddagger}^{\ddagger}$ ORCID: orcid.org/0000-0003-3501-4608. Department of Data Science and Knowledge Engineering, Maastricht University, The Netherlands.

${ }^{\S}$ Institute of Informatics, University of Warsaw, Poland, kfleszar@mimuw.edu.pl ORCID: orcid.org/0000-0002-1129-3289. K. Fleszar was supported by ERC consolidator grant no. 772346.

"ORCID: orcid.org/0000-0001-7833-0454. F. Lipp was supported by a Cusanuswerk PhD scholarship.

"Pidstryhach Institute for Applied Problems of Mechanics and Mathematics, National Academy of Sciences of Ukraine, Lviv, Ukraine, alexander.ravsky@uni-wuerzburg.de

** Institut für Informatik, Humboldt-Universität zu Berlin, Germany, verbitsk@informatik.hu-berlin.de. On leave from the IAPMM, Lviv, Ukraine. O. Verbitsky was supported by DFG grant VE 652/1-2.

${ }^{\dagger \dagger}$ ORCID: orcid.org/0000-0001-5872-718X. A. Wolff was supported by DFG grant WO 758/9-1. 


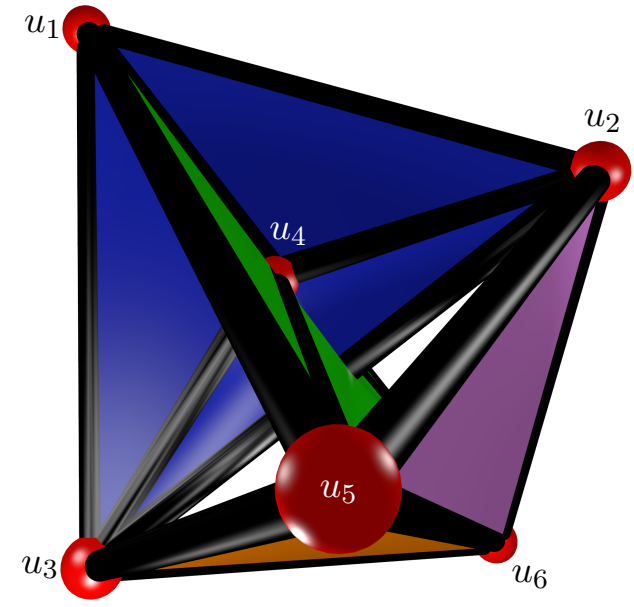

Figure 1. $K_{6}$ can be drawn straight-line and crossing-free on four planes. This is optimal, that is, $\rho_{3}^{2}\left(K_{6}\right)=4$.

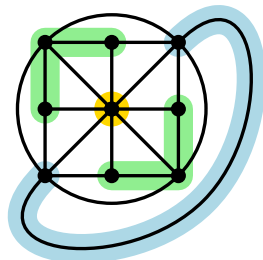

(a)

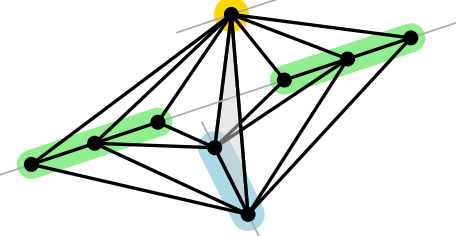

(b)
Figure 2. (a) Planar 9-vertex graph $G$ with $\pi_{3}^{1}(G)=3$ and (b) a $3 \mathrm{D}$ drawing of $G$ on three lines.

planes do we need for a given graph? For example, $K_{6}$ needs four planes; see Fig. $1 .{ }^{1}$ Note that this question is different from the well-known concept of a book embedding where all vertices lie on one line (the spine) and edges lie on a limited number of adjacent half-planes (the pages). In contrast, we put no restriction on the mutual position of planes, the vertices can be located in the planes arbitrarily, and the edges must be straight-line.

In a weaker setting, we require only the vertices to be located on a limited number of planes (or lines). For example, the planar graph with nine vertices shown in Fig. 2 can be drawn in 3D such that its vertices are contained in three lines. It turns out that every planar graph with at most eight vertices can be drawn in 3D such that its vertices lie on two lines, see Remark 3.2. This version of our problem is related to the well-studied problem of drawing a graph straight-line in a 3D grid of bounded volume [36,86]: If a graph can be drawn with all vertices on a grid of volume $v$, then $v^{1 / 3}$ planes or $v^{2 / 3}$ lines suffice to cover all vertices. We now formalize the problem.

Definition 1.1. Let $1 \leq l<d$, and let $G$ be a graph. We define the $l$-dimensional affine cover number of $G$ in $\mathbb{R}^{d}$, denoted by $\rho_{d}^{l}(G)$, as the minimum number of $l$-dimensional planes in $\mathbb{R}^{d}$ such that $G$ has a crossing-free straight-line drawing that is contained in the union of these planes. We define $\pi_{d}^{l}(G)$, the weak l-dimensional affine cover number of $G$ in $\mathbb{R}^{d}$, similarly to $\rho_{d}^{l}(G)$, but under the weaker restriction that the vertices (and not necessarily the edges) of $G$ are contained in the union of the planes. Finally, the parallel affine cover number, $\bar{\pi}_{d}^{l}(G)$, is a restricted version of $\pi_{d}^{l}(G)$, in which we insist that the planes are parallel. The one-dimensional affine cover numbers $\rho_{2}^{1}(G), \pi_{2}^{1}(G)$, and $\bar{\pi}_{2}^{1}(G)$ in 2D are only defined if the graph $G$ is planar.

\footnotetext{
${ }^{1}$ An interactive and 3D-printable version of this model is available as supplementary material on the JoCG webpage and on our webpage: http://www1.pub.informatik. uni-wuerzburg.de/pub/data/rho/
} 
Clearly, for any combination of $l$ and $d$, we have $\pi_{d}^{l}(G) \leq \bar{\pi}_{d}^{l}(G)$ and $\pi_{d}^{l}(G) \leq \rho_{d}^{l}(G)$. Larger values of $l$ and $d$ give us more freedom for drawing graphs and, therefore, smaller $\pi$ and $\rho$-values. Formally, for any graph $G$, if $l^{\prime} \leq l$ and $d^{\prime} \leq d$, then we have $\pi_{d}^{l}(G) \leq \pi_{d^{\prime}}^{l^{\prime}}(G)$, $\rho_{d}^{l}(G) \leq \rho_{d^{\prime}}^{l^{\prime}}(G)$, and $\bar{\pi}_{d}^{l}(G) \leq \bar{\pi}_{d^{\prime}}^{l^{\prime}}(G)$. But in most cases this freedom is not essential. For example, it suffices to consider $l \leq 2$ because otherwise $\rho_{d}^{l}(G)=1$. Less obviously, we can actually focus on $d \leq 3$ because every graph can be drawn in 3-space as effectively as in high dimensional spaces. This fact is formalized in the following theorem, whose proof is given at the end of this section.

Theorem 1.2. For any integers $1 \leq l<d, d \geq 3$, and for any graph $G$, it holds that $\pi_{d}^{l}(G)=\pi_{3}^{l}(G), \bar{\pi}_{d}^{l}(G)=\bar{\pi}_{3}^{l}(G)$, and $\rho_{d}^{l}(G)=\rho_{3}^{l}(G)$.

Thus, our task is to investigate the cases $1 \leq l<d \leq 3$. We call $\rho_{2}^{1}(G)$ and $\rho_{3}^{1}(G)$ the line cover numbers in $2 \mathrm{D}$ and $3 \mathrm{D}, \rho_{3}^{2}(G)$ the plane cover number, and analogously for the weak and parallel versions.

Related work. We have already briefly mentioned 3D graph drawing on the grid, a topic that has been surveyed by Wood [86] and by Dujmović and Whitesides [36]. For example, Dujmović [28], improving on a result of Di Battista et al. [25], showed that any planar graph can be drawn into a 3D-grid of volume $O(n \log n)$. Recently, Dujmović et al. [31] improved on this result by showing that even a grid of volume $O(n)$ is sufficient.

An interesting variant of our problem is to study drawings whose edge sets are represented (or covered) by as few objects as possible. The type of objects that have been used are straight-line segments [29,38], circular arcs [77], and circles or spheres [61]. The idea behind this objective is to keep the visual complexity of a drawing low for the observer. Jamison [56] studied the slope number of $K_{n}$; given a graph $G$, the slope number, slope $(G)$, is the minimum number of distinct slopes in a straight-line drawing of $G$. As a variant, Dujmović et al. [29] introduced the planar slope number for a planar graph $G$, as the smallest number of slopes among all planar drawings of $G$. They also introduced the segment number of a planar graph $G, \operatorname{seg}(G)$, as the smallest number of line segments whose union contains a planar straight-line drawing of $G$. All three numbers have been studied extensively since then $[53,55,57,65]$.

Durocher et al. [39] showed that it is NP-hard to compute the segment number, even for planar graphs of maximum degree 4 . They pose it as an open problem to study what they call minimum-line drawings, that is, minimum segment-drawings that additionally minimize the number of lines needed to cover them. This is not the same as directly minimizing the number of lines.

Further, Dujmovic et al. [29] asked whether there is some universal function $f$ such that, for any graph $G$, slope $(G) \leq f(\Delta(G))$, independently of the size of $G$. Their question was answered to the negative by Pach and Pálvögyi [66] and, independently, by Barát et al. [4]. Pach and Pálvögyi [66] showed that for any sufficiently large integer $n$ and $\Delta \geq 5$, there is an $n$-vertex graph $G$ of maximum degree $\Delta$ whose slope number is larger than $n^{1 / 2-O(1 / \Delta)}$. This bound was later improved to $n^{1-O(1 / \Delta)}$ by Dujmović et al. [35].

Schulz [77] showed that using circular arcs instead of line segments gives better 


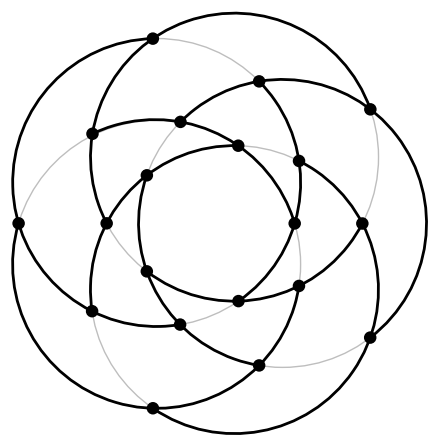

(a) $10 \operatorname{arcs} / 5$ circles [61].

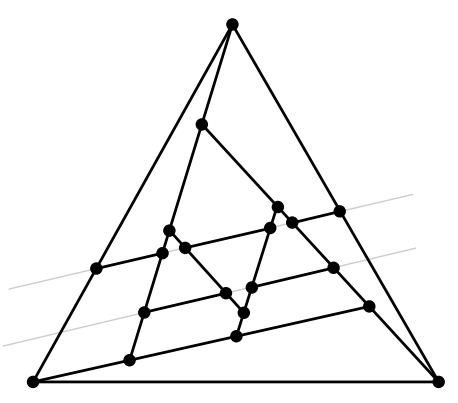

(b) 13 segments / 10 lines [75].

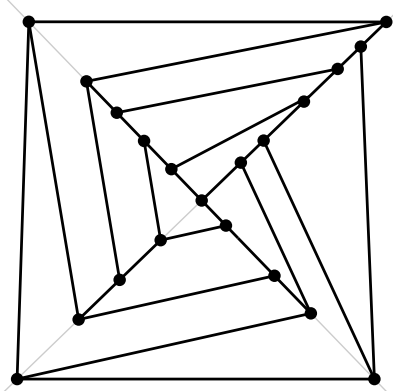

(c) weak line cover [43].

Figure 3. Drawings of the dodecahedron using as few objects as possible.

bounds or permits drawings on a smaller grid. As an example, he drew the dodecahedron on 10 arcs, which is optimal. Kryven et al. [61] modified Schulz's drawing so that the 10 arcs are covered by only 5 circles (see Fig. 3a). Scherm [75] constructed a drawing of the dodecahedron on 13 line segments and 10 lines, which is depicted in Fig. 3b. Kryven et al. [61] showed that these drawings are (close to) optimal; for the dodecahedron, they proved a lower bound of 13 for the segment number and a lower bound of 9 for the line cover number (in 2D and 3D). Firman et al. [43] computed the weak line cover numbers of the Platonic graphs; in particular, the weak line cover number of the dodecahedron is 2 ; see Fig. 3c.

Hültenschmidt et al. [54] studied the visual complexity of drawings of planar graphs. For example, they showed upper bounds for the number of segments and arcs in drawings of trees, triangulations, and general planar graphs. Kindermann et al. [58] also conducted a user study to evaluate the benefits of drawings with low visual complexity.

Classes of planar graphs with bounded $\pi_{2}^{1}$-value are of interest in the context of untangling $[11,46,67]$. If a graph $G$ has $n$ vertices and $\pi_{2}^{1}(G) \leq c$, then $G$ admits a straightline drawing with at least $n / c$ collinear vertices. Large collinear sets are important because if $G$ admits a set of $k$ collinear vertices, then any straight-line drawing of $G$ can be untangled while at least $\sqrt{k}$ vertices remain fixed [30]; see also [22,32,72].

In subsequent work, Eppstein [40] built a cubic 3-connected bipartite planar graph $G_{\ell}$ with $n=O\left(\ell^{3}\right)$ vertices and $\pi_{2}^{1}(G) \geq \ell$ (which improves our Theorem 3.4 where we show that, for infinitely many values of $n$, there is an $n$-vertex triangulation $G$ with $\Delta(G) \leq 12$ and $\left.\pi_{2}^{1}(G) \geq n^{0.01}\right)$. Eppstein also constructed, for any $k>0$, a series-parallel graph $G$ with $\pi_{2}^{1}(G)>k$ and an apex-tree $T$ (a graph formed by adding one vertex to a tree) with $\pi_{2}^{1}(T)>k$. On the positive side, he proved that, for every $n$-vertex apex-tree $T$, we have $\bar{\pi}_{2}^{1}(T) \in O(\log n)$. Felsner [41] showed that $\pi_{2}^{1}(G)<\sqrt{2 n}$ for any 4-connected planar graph $G$ with $n$ vertices. He also showed that Eppstein's graph $G_{\ell}$ can be turned into a 4 -connected triangulation by adding a small number of vertices and many edges. Hence, this class contains graphs $G$ with $\pi_{2}^{1}(G) \in \Omega\left(n^{1 / 3}\right)$. Biedl et al. [7] showed that the universal stacked triangulation of depth $d, G_{d}$, has $\pi_{2}^{1}\left(G_{d}\right)=d+1$. Concerning $3 \mathrm{D}$, they showed that any $n$-vertex graph $G$ with $\rho_{3}^{2}(G)=2$ has at most $5 n-19$ edges, which is tight. 
Our contribution. Our research goes into three directions.

First, we show lower and upper bounds for the affine cover numbers of graphs in special graph classes, such as the complete graphs and the complete bipartite graphs. Most versions of the affine cover numbers of these graphs can be determined easily, except one case which is much more subtle: We determine $\rho_{3}^{2}\left(K_{n}\right)$ only asymptotically, up to a factor of 2 (see Theorem 2.16). We also compute the exact values of $\rho_{3}^{2}\left(K_{n}\right)$ for small $n$ (see Theorem 2.19 and Table 1). As other results in this direction, we prove that almost any $n$ vertex cubic graph $G$ has $\rho_{3}^{1}(G)>n / 5$ (Theorem 2.9) and that any almost-planar graph (a graph that becomes planar after the deletion of a single edge) can be drawn straight-line on the union of four planes (Theorem 2.14).

Second, we relate the affine cover numbers to standard combinatorial characteristics of graphs and to parameters that have been studied in graph drawing. In Sections 2.1 and 2.2, we characterize $\pi_{3}^{1}(G)$ and $\pi_{3}^{2}(G)$ in terms of the linear vertex arboricity and the vertex thickness, respectively. This characterization implies that both $\pi_{3}^{1}(G)$ and $\pi_{3}^{2}(G)$ are linearly related to the chromatic number of the graph $G$. We also prove that any graph $G$ has balanced separators of size at most $\rho_{3}^{1}(G)$ and conclude from this that $\rho_{3}^{1}(G) \geq \operatorname{tw}(G) / 3$, where $\operatorname{tw}(G)$ denotes the treewidth of $G$ (Theorem 2.11; for the definition of treewidth, see [27]). In Section 3.3, we analyze the relationship between $\rho_{2}^{1}(G)$ and the above-mentioned segment number $\operatorname{seg}(G)$ of a graph (see Table 2). We prove that $\operatorname{seg}(G)=O\left(\rho_{2}^{1}(G)^{2}\right)$ for any connected graph $G$ and show that this bound is optimal (see Lemma 3.9 and Example 3.8).

Third, we pay special attention to planar graphs (Section 3). Among other results, we construct planar graphs with a large gap between the parameters $\rho_{3}^{1}(G)$ and $\rho_{2}^{1}(G)$ (see Theorem 3.13).

We also investigate the parallel affine cover numbers $\bar{\pi}_{2}^{1}$ and $\bar{\pi}_{3}^{1}$ (Section 4). In fact, $\bar{\pi}_{3}^{1}(G)$ equals the improper track number of $G$, which was introduced by Dujmović et al. [33]. We discuss some consequences of known results on this parameter and also notice relations between $\bar{\pi}_{3}^{1}(G)$ and other parameters under consideration, namely $\rho_{3}^{2}(G)$ and $\pi_{2}^{1}(G)$.

Remark on the computational complexity. In a companion paper [18], we investigate the computational complexity of computing the $\rho$ - and $\pi$-numbers. We argue that it is NP-hard to decide whether a given graph has a $\pi_{3}^{1}$ - or $\pi_{3}^{2}$-value of 2 and that both values are even hard to approximate. This result is based on Theorems 2.1 and 2.4 and Corollaries 2.2 and 2.5 in the present paper. For the missing parameter $\pi_{2}^{1}$, Biedl et al. [7] showed that it is NP-hard to decide, for a given graph $G$, whether $\pi_{2}^{1}(G)=2$. While the graphs with a $\rho_{3}^{2}$-value of 1 are exactly the planar graphs (and hence, can be recognized in linear time), it turns out that recognizing graphs with a $\rho_{3}^{2}$-value of 2 is already NP-hard [18]. In contrast to this, the problems of deciding whether $\rho_{3}^{1}(G) \leq k$ or $\rho_{2}^{1}(G) \leq k$ are solvable in polynomial time for each fixed $k$. However, the versions of these problems with $k$ being part of the input are complete for the complexity class $\exists \mathbb{R}$ which is based on the existential theory of the reals, which plays an important role in computational geometry [74].

Proof of Theorem 1.2. We prove the following statement, which implies the theorem: 
$\left.{ }^{*}\right)$ For any finite family $\mathcal{L}$ of lines in $\mathbb{R}^{d}$, there exists a linear transformation $A: \mathbb{R}^{d} \rightarrow \mathbb{R}^{3}$ that is injective on $L_{0}=\bigcup\{\ell: \ell \in \mathcal{L}\}$, the set of all points of the lines in $\mathcal{L}$.

To obtain the theorem from here, we set $\mathcal{L}$ to be a family of lines covering the edges or, respectively, the vertices of a given $d$-dimensional drawing of $G$. Then $A$ transforms this drawing into a 3-dimensional drawing of $G$, which is covered by the set of lines $\{A(\ell): \ell \in \mathcal{L}\}$. This immediately gives us the case $l=1$ of the theorem. For the case of $l=2$, consider a set $\mathcal{P}$ of 2 -dimensional planes covering the $d$-dimensional drawing. Then the 3 -dimensional drawing is covered by $\{A(p): p \in \mathcal{P}\}$. If it happens that some $A(p)$ is a line or a point, it can be arbitrarily extended to a 2 -dimensional plane in $\mathbb{R}^{3}$.

We now prove statement $(*)$ by induction on $d$. If $d=3$, we let $A$ be the identity map on $\mathbb{R}^{3}$. Suppose that $d>3$.

Regarding two lines $\ell$ and $\ell^{\prime}$ in $\mathbb{R}^{d}$ as 1 -dimensional affine subspaces, we consider their Minkowski difference $\ell-\ell^{\prime}=\left\{x-x^{\prime}: x \in \ell, x^{\prime} \in \ell^{\prime}\right\}$. Note that $\ell-\ell^{\prime}$ is contained in a plane, that is, in a 2 -dimensional affine subspace of $\mathbb{R}^{d}$. Denote $L=\bigcup\left\{\ell-\ell^{\prime}: \ell, \ell^{\prime} \in \mathcal{L}\right\}$. Let $L^{\prime}=\{t x: t \in \mathbb{R}, x \in L\}$ be the union of all lines going through the origin 0 of $\mathbb{R}^{d}$ and intersecting the set $L$. Since the set $L$ is contained in a union of finitely many planes in the space $\mathbb{R}^{d}$, the set $L^{\prime}$ is contained in a union of finitely many 3-dimensional linear subspaces of $\mathbb{R}^{d}$ (each of them contains the origin 0 ). Since $d>3$, there exists a line $\ell_{0} \ni 0$ such that $\ell_{0} \cap L=\{0\}$. Now, let $A_{0}: \mathbb{R}^{d} \rightarrow \mathbb{R}^{d-1}$ be an arbitrary linear transformation such that ker $A_{0}:=\left\{x \in \mathbb{R}^{d}: A_{0} x=0\right\}=\ell_{0}$. Let $x, y \in L_{0}$ be arbitrary points such that $A_{0} x=A_{0} y$. Observe that $x-y \in L \cap \operatorname{ker} A_{0}=L \cap \ell_{0}=\{0\}$ holds, so $x=y$. Thus, the map $A_{0}$ is injective on $L_{0}$ and so the family of lines $\left\{A_{0} \ell: \ell \in \mathcal{L}\right\}$ is contained in $\mathbb{R}^{d-1}$ and satisfies the assumption of the statement for dimension $d-1$. By the inductive assumption applied to the family, there exists a linear transformation $A_{1}: \mathbb{R}^{d-1} \rightarrow \mathbb{R}^{3}$ injective on the union of all $\ell \in \mathcal{L}$. The composition $A=A_{1} A_{0}$ of $A_{1}$ and $A_{0}$ is the transformation needed for statement $(*)$.

Notation and terminology. For a graph $G$, we use $n$ and $m$ to denote the numbers of vertices and edges of $G$, respectively. Let $\Delta(G)=\max _{v \in V} \operatorname{deg} v$ denote the maximum degree of a vertex in $G$. Furthermore, we will use the standard notation $\chi(G)$ for the chromatic number, $\operatorname{tw}(G)$ for the treewidth, and $\operatorname{diam}(G)$ for the diameter of $G$. For a graph $G$, let $V(G)$ be the vertex set of $G$ and let $E(G)$ be the edge set of $G$. The Cartesian product of graphs $G$ and $H$ is denoted by $G \times H$. Let $K_{n}$ denote the complete graph on $n$ vertices and let $K_{p, q}$ denote the complete bipartite graph where the two parts of the graph have $p$ respectively $q$ vertices. For a graph $G$ and a set $V^{\prime} \subseteq V(G)$, we use $G\left[V^{\prime}\right]$ to denote the subgraph of $G$ induced by $V^{\prime}$.

Cubic graphs are graphs where all vertices have degree 3. Recall also that a graph is $k$-connected if it has more than $k$ vertices and stays connected after removal of any set of up to $(k-1)$ vertices. A planar graph $G$ is maximal if adding an edge between any two non-adjacent vertices of $G$ violates planarity. Maximal planar graphs on more than three vertices are 3-connected. Clearly, all facial cycles in such graphs have length 3. By this reason, maximal planar graphs are also called triangulations, and we use the last term throughout the paper. 
By a $3 D$ grid drawing of a graph $G$ we mean a straight-line drawing of $G$ whose vertices are located at points of the integer grid $\mathbb{Z}^{3}$. The bounding box of a $3 \mathrm{D}$ grid drawing is the inclusion-wise smallest axis-aligned cuboid that contains the drawing. If the bounding box has sides of lengths $a-1, b-1$, and $c-1$, we say that the $3 \mathrm{D}$ grid drawing is of size $a \times b \times c$. The volume of this drawing is $a b c$, which is the number of grid points contained in the bounding box. A $2 D$ grid drawing of a planar graph and its area are defined similarly.

\section{The Affine Cover Numbers of General Graphs in 3-Space}

In the following sections we study the affine cover numbers of general graphs. We start with the weak line cover number and the weak plane cover number and relate those to the graph parameters linear vertex arboricity, vertex thickness, and chromatic number. After that, we show lower bounds for the line cover number in terms of the number of vertices and in terms of treewidth. Finally, we consider the plane cover number of almost-planar graphs. At the end of each section, we examine the values of the affine cover numbers for complete (bipartite) graphs.

\subsection{Placing Vertices on Few Lines $\left(\pi_{3}^{1}\right)$}

A linear forest is a forest whose connected components are paths. The linear vertex arboricity $\operatorname{lva}(G)$ of a graph $G$ equals the smallest size $r$ of a partition $V(G)=V_{1} \cup \cdots \cup V_{r}$ such that every $V_{i}$ induces a linear forest (Matsumoto [62]). This notion, which is an induced version of the fruitful concept of linear arboricity (see Remark 2.8 below), appears very relevant to our topic. The following result is based on a construction of Pach et al. [68]. For the reader's convenience, we give some details of this construction in the proof.

Theorem 2.1. For any graph $G$, it holds that $\pi_{3}^{1}(G)=\operatorname{lva}(G)$. Moreover, $G$ has a $3 D$ grid drawing of size $r \times 4 r n \times 4 r^{2} n$, where $r=\operatorname{lva}(G)$, with the vertices covered by $r$ lines in $\mathbb{R}^{3}$.

Proof. The inequality $\operatorname{lva}(G) \leq \pi_{3}^{1}(G)$ holds because any set of collinear vertices induces a linear forest in $G$. We, therefore, have to prove the converse inequality $\pi_{3}^{1}(G) \leq \operatorname{lva}(G)$.

Let $K(r, n)$ denote the complete $r$-partite graph with each of the $r$ vertex classes having size $n$. Pach et al. [68] show that $K(r, n)$ admits a 3D grid drawing of size $r \times 4 r n \times 4 r^{2} n$. They construct this drawing as follows. Let $p$ be the smallest prime such that $p \geq 2 r-1$ and set $N=p n$. By Bertrand's postulate, $p<4 r$ and, hence, $N<4 r n$. Given $0 \leq i \leq r-1$, consider $U_{i}=\left\{(i, t, i t): 0 \leq t<N, t \equiv i^{2}(\bmod p)\right\}$. These $r$ sets are pairwise disjoint, and each of them has precisely $N / p=n$ elements. Connect any two points belonging to different $U_{i}$ 's by a straight-line segment. Pach et al. [68, page 48] showed that no two segments in this drawing of $K(r, n)$ cross each other.

Note that $U_{i}$ is contained in the line $\ell_{i}=\{(i, 0,0)+t(0,1, i): t \in \mathbb{R}\}$. We will use two additional properties of the above construction:

(i) The lines $\ell_{i}$ and $\ell_{j}$ for any $i \neq j$ do not intersect as they lie in different planes parallel to the $y z$-plane. 
(ii) There is also no crossing between any edge of $K(r, n)$ and any line $\ell_{i}$. This fact can be derived from Case 2 of the proof by Pach et al. [68, page 49].

Now, let $V(G)=V_{1} \cup \cdots \cup V_{r}$ be a partition of the vertex set of $G$ where each $G\left[V_{i}\right]$ is a linear forest. Fix a map $f: V(G) \rightarrow \mathbb{Z}^{3}$ such that, for each $i, f$ maps $V_{i}$ into $U_{i}$ and the restriction of $f$ to $V_{i}$ induces a crossing-free drawing of the linear forest $G\left[V_{i}\right]$ on the line $\ell_{i}$. Note that $f$ induces a crossing-free straight-line drawing of the whole graph $G$. Indeed, as we have already mentioned, there are no crossings between those edges of $G$ which are also edges of the underlying drawing of $K(r, n)$. The edges of different linear forests $G\left[V_{i}\right]$ and $G\left[V_{j}\right]$ do not cross by Property (i). Finally, if one edge belongs to one of the linear forests $G\left[V_{i}\right]$ and another does not, they do not cross by Property (ii). It remains to note that the bounding box of this drawing of $G$ is included in the bounding box of the underlying drawing of $K(r, n)$.

Corollary 2.2. For any graph $G, \chi(G) / 2 \leq \pi_{3}^{1}(G) \leq \chi(G)$.

Proof. We have lva $(G) \leq \chi(G)$ because any independent set is a linear forest. On the other hand, $\chi(G) \leq 2 \operatorname{lva}(G)$ because any linear forest is 2 -colorable.

The lower bound in this corollary is tight for complete graphs $K_{n}$ with even $n$, see Example 2.3(a). The upper bound is tight for complete $r$-partite graphs (with sufficiently many vertices in each set of the partition). Since $\chi(G) \leq \Delta(G)+1$ by Brooks' theorem [13], Corollary 2.2 readily implies that $\pi_{3}^{1}(G) \leq \Delta(G)+1$. This can be considerably improved using a relationship between the linear vertex arboricity and the maximum degree that is established by Matsumoto [62]. Matsumoto's result implies that $\pi_{3}^{1}(G) \leq \Delta(G) / 2+1$ for any connected graph $G$. Moreover, if $\Delta(G)=2 d$, then $\pi_{3}^{1}(G)=d+1$ if and only if $G$ is a cycle or the complete graph $K_{2 d+1}$.

Example 2.3. The weak line cover number of complete (bipartite) graphs is as follows.

(a) $\pi_{3}^{1}\left(K_{n}\right)=\lceil n / 2\rceil$ for any $n \geq 2$; therefore, $\pi_{3}^{1}(G) \leq\lceil n / 2\rceil$ for every graph $G$.

(b) $\pi_{3}^{1}\left(K_{p, q}\right)=2$ for any $1 \leq p \leq q$; except for $\pi_{3}^{1}\left(K_{1,1}\right)=\pi_{3}^{1}\left(K_{1,2}\right)=1$.

Proof. The lower bound for $\pi_{3}^{1}\left(K_{n}\right)$ and the upper bound for $\pi_{3}^{1}\left(K_{p, q}\right)$ follow from Corollary 2.2. The upper bound $\pi_{3}^{1}\left(K_{n}\right) \leq\lceil n / 2\rceil$ is given by any 3-dimensional drawing of $K_{n}$; we can split the vertices in pairs and draw a line through each pair.

\subsection{Placing Vertices on Few Planes $\left(\pi_{3}^{2}\right)$}

We now turn to the weak plane cover numbers. The vertex thickness $\operatorname{vt}(G)$ of a graph $G$ is the smallest size $r$ of a partition $V(G)=V_{1} \cup \cdots \cup V_{r}$ such that the induced subgraphs $G\left[V_{1}\right], \ldots, G\left[V_{r}\right]$ are all planar.

Theorem 2.4. For any graph $G$, it holds that $\pi_{3}^{2}(G)=\bar{\pi}_{3}^{2}(G)=\operatorname{vt}(G)$. Moreover, $G$ has a $3 D$ grid drawing of size $\operatorname{vt}(G) \times O\left(m^{2}\right) \times O(m \cdot \max \{n, m\}$ ) (whose vertices are obviously covered by $\operatorname{vt}(G)$ planes), where $m$ is the number of edges of $G$ and $n$ is the number of vertices of $G$. 
Proof. As the relations $\operatorname{vt}(G) \leq \pi_{3}^{2}(G) \leq \bar{\pi}_{3}^{2}(G)$ are obvious, it remains to prove the inequality $\bar{\pi}_{3}^{2}(G) \leq \operatorname{vt}(G)$. Since this inequality follows from the existence of a drawing of the size specified in the theorem statement, it suffices to prove that such a drawing exists. If $G$ contains isolated vertices, we ignore them at first and show that the resulting graph can be drawn in a 3D grid of size $\operatorname{vt}(G) \times O\left(\mathrm{~m}^{2}\right) \times O\left(\mathrm{~m}^{2}\right)$. Obviously, we can reinsert these isolated vertices into this drawing by adding $O(n)$ grid points. In the following, we assume that there are no isolated vertices in $G$ and, hence, $n \leq 2 m$.

Let $r=\operatorname{vt}(G)$ and let $V_{1}, \ldots, V_{r}$ be a partition of the vertex set of $G$ such that each $G\left[V_{i}\right]$ is a planar graph. As well known, every planar graph admits a plane straight-line drawing on an $O(n) \times O(n)$ grid $[24,76]$. Let us fix such a drawing $\delta_{i}$ for each $G\left[V_{i}\right]$ and place it in the plane $z=i$. An edge $u v$ is called horizontal if both $u$ and $v$ belong to the same $V_{i}$ for some $i \leq r$. We now have to resolve two problems:

- A non-horizontal edge can pass through a vertex of some $\delta_{i}$;

- Two edges that are not both horizontal can cross each other.

In order to remove all possible crossings, we replace each $\delta_{i}$ with its random perturbation $\delta_{i}^{\prime}$ (still in the same plane $z=i$ ) and prove that, with non-zero probability, no crossing occurs.

Specifically, let $s$ and $t$ be parameters that will be chosen later. Let $T_{a, b, p, q}$ be an affine tranformation of the $x y$-plane defined by

$$
T_{a, b, p, q}\left(\begin{array}{c}
x \\
y
\end{array}\right)=\left(\begin{array}{cc}
a & -b \\
b & a
\end{array}\right)\left(\begin{array}{l}
x \\
y
\end{array}\right)+\left(\begin{array}{l}
p \\
q
\end{array}\right)
$$

where $a, b, p$, and $q$ are integers such that $0 \leq p, q<s, 1 \leq b<a \leq t$, and $a$ and $b$ are coprime. Note that $T_{a, b, p, q}$ consists of a dilating rotation followed by a shift, and that it transforms integral points into integral points. The random drawings $\delta_{i}^{\prime}$ are obtained by choosing $a, b, p$, and $q$ at random and applying $T_{a, b, p, q}$ to $\delta_{i}$ (this is done independently for different $i \leq r)$. Note that the resulting 3D grid drawing has size $r \times O(t n+s) \times O(t n+s)$.

For each fixed edge $u v$ and vertex $w$ such that $u \in V_{i}, w \in V_{j}$, and $v \in V_{k}$ for some $i<j<k$, let us estimate the probability that $u v$ passes through $w$ in the drawing. Conditioned on the positions of $\delta_{l}^{\prime}$ for all $l \neq j$ and on the choice of the parameters $a$ and $b$ in $T_{a, b, p, q}$ for $\delta_{j}^{\prime}$, this probability is clearly at most $1 / s^{2}$. Therefore, this probability is at most $1 / s^{2}$ also if all $\delta_{l}^{\prime}$ are chosen at random. It follows that there is a non-horizontal edge passing through some vertex with probability at most $\mathrm{mn} / \mathrm{s}^{2}$.

Consider now two edges $u_{1} v_{1}$ and $u_{2} v_{2}$. If there is an $i \leq r$ such that $V_{i}$ contains exactly one of the vertices $u_{1}, v_{1}, u_{2}$, and $v_{2}$, then an argument similar to the above shows that $u_{1} v_{1}$ and $u_{2} v_{2}$ cross with probability at most $1 / \mathrm{s}$. It follows that some edges of this kind cross each other with probability at most $\mathrm{m}^{2} / \mathrm{s}$.

Suppose now that $u_{1}, u_{2} \in V_{i}$ and $v_{1}, v_{2} \in V_{j}$. Note that shifts cannot resolve the possible crossing of the edges $u_{1} v_{1}$ and $u_{2} v_{2}$. Luckily, if we fix $\delta_{i}^{\prime}$ and "rotate" $\delta_{j}^{\prime}$ by means of $T_{a, b, p, q}$ with random $a, b$ and fixed $p, q$, then $u_{1} v_{1}$ and $u_{2} v_{2}$ will cross for at most one choice of the pair $a, b$. The probability of this event is bounded by $O\left(1 / t^{2}\right)$ because the number of coprime $a$ and $b$ such that $1 \leq b<a \leq t$ is known to be asymptotically $\frac{3}{\pi^{2}} t^{2}+O(t \log t)$ [48]. It follows that some edges of this kind cross each other with probability at most $O\left(\mathrm{~m}^{2} / t^{2}\right)$. 
Summarizing, we see that the random drawing of $G$ will have a crossing with probability bounded by

$$
\frac{m n}{s^{2}}+\frac{m^{2}}{s}+O\left(\frac{m^{2}}{t^{2}}\right)
$$

This probability can be ensured to be strictly smaller than 1 by choosing large enough parameters $s=O\left(\mathrm{~m}^{2}\right)$ and $t=O(\mathrm{~m})$. We conclude that for such choice of $s$ and $t$ there is at least one crossing-free drawing. Since $O(t n+s)=O\left(m n+m^{2}\right)=O\left(m^{2}\right)$, such a drawing has a size of $r \times O\left(m^{2}\right) \times O\left(m^{2}\right)$.

Corollary 2.5. For any graph $G, \chi(G) / 4 \leq \pi_{3}^{2}(G) \leq \chi(G)$.

Proof. These inequalities follow from the relations vt $(G) \leq \chi(G)$ (any independent set is a planar subgraph) and $\chi(G) \leq 4 \operatorname{vt}(G)$ (a straightforward consequence of the four color theorem).

Again (similar to Corollary 2.2), the lower bound is tight for complete graphs $K_{n}$ with $n$ being a multiple of 4, see Example 2.6(a); the upper bound is tight for complete $r$-partite graphs with sufficiently many vertices in each set of the partition.

Example 2.6. The weak plane cover number of complete (bipartite) graphs is as follows.

(a) $\pi_{3}^{2}\left(K_{n}\right)=\lceil n / 4\rceil$ for any $n \geq 2$; therefore, $\pi_{3}^{2}(G) \leq\lceil n / 4\rceil$ for every graph $G$.

(b) $\pi_{3}^{2}\left(K_{p, q}\right)=2$ for any $3 \leq p \leq q$ and $\pi_{3}^{2}\left(K_{p, q}\right)=1$ for any $p \leq 2$ and $q \geq p$.

Proof. (a) By Theorem 2.4, $\pi_{3}^{2}\left(K_{n}\right)$ is equal to the smallest size $r$ of a partition $V(G)=$ $V_{1} \cup \cdots \cup V_{r}$ where each $V_{i}$ induces a planar subgraph of $K_{n}$, that is, has size at most 4 (as $K_{4}$ is planar and $K_{5}$ is not). Such a partition exists if and only if $r \geq\lceil n / 4\rceil$.

(b) The result for the planar complete bipartite graphs is trivial. So consider $K_{p, q}$ with $3 \leq p \leq q$. Obviously, the graph is non-planar and thus its vertices cannot be placed on one plane. The upper bound follows by Corollary 2.5.

\subsection{Placing Edges on Few Lines $\left(\rho_{3}^{1}\right)$}

This section contains lower bounds for $\rho_{3}^{1}(G)$ in terms of different combinatorial characteristics of a graph $G$.

We call a vertex $v$ of a graph $G$ essential if $\operatorname{deg} v \geq 3$ or if $v$ belongs to a $K_{3}$ subgraph of $G$. We denote the number of essential vertices in $G$ by $\operatorname{es}(G)$.

Lemma 2.7. The following bounds hold for any graph $G$.

(a) $\rho_{3}^{1}(G) \geq(1+\sqrt{1+8 \operatorname{es}(G)}) / 2>\sqrt{2 \operatorname{es}(G)}$.

(b) $\rho_{3}^{1}(G)>\sqrt{m^{2} / n-m}$ if $n=|V(G)| \geq 1$ and $m=|E(G)| \geq n$.

Proof. (a) In any drawing of a graph $G$, any essential vertex is shared by two edges not lying on the same line. Therefore, each such vertex is an intersection point of at least two lines, which implies that $\operatorname{es}(G) \leq\left(\begin{array}{c}\rho_{3}^{1}(G) \\ 2\end{array}\right)$. Hence, $\rho_{3}^{1}(G) \geq(1+\sqrt{1+8 \operatorname{es}(G)}) / 2>\sqrt{2 \operatorname{es}(G)}$. 
(b) Each vertex $v$ serves as an intersection point for at least $\lceil\operatorname{deg} v / 2\rceil(\lceil\operatorname{deg} v / 2\rceil-1) / 2$ pairs of lines. This implies that

$$
\begin{aligned}
\left(\begin{array}{c}
\rho_{3}^{1}(G) \\
2
\end{array}\right) & \geq \frac{1}{2} \sum_{v \in V(G)}\left\lceil\frac{\operatorname{deg} v}{2}\right\rceil\left(\left\lceil\frac{\operatorname{deg} v}{2}\right\rceil-1\right) \geq \sum \frac{\operatorname{deg} v(\operatorname{deg} v-2)}{8}= \\
& =\frac{1}{8} \sum(\operatorname{deg} v)^{2}-\frac{1}{4} \sum \operatorname{deg} v \geq \frac{1}{8 n}\left(\sum \operatorname{deg} v\right)^{2}-\frac{1}{4} 2 m=\frac{m^{2}}{2 n}-\frac{m}{2} .
\end{aligned}
$$

The last inequality follows by the inequality between arithmetic and quadratic means. Hence, $\rho_{3}^{1}(G)>\sqrt{m^{2} / n-m}$.

Part (a) of Lemma 2.7 implies that $\rho_{3}^{1}(G)>\sqrt{2 n}$ if a graph $G$ has no vertices of degree 1 and 2, while part (b) yields $\rho_{3}^{1}(G)>\sqrt{m / 2}$ for all such $G$. Note that the graph $k C_{4}$, the disjoint union of $k$ 4-cycles, has no essential vertex, but each cycle will need at least 3 intersection points of lines, implying $\rho_{3}^{1}\left(k C_{4}\right)>\sqrt{6 k}$. Thus, $\rho_{3}^{1}(G)$ cannot be bounded from above in terms of es $(G)$.

Remark 2.8. The linear arboricity $\mathrm{la}(G)$ of a graph $G$ is the minimum number of linear forests which partition the edge set of $G$ [51]. Clearly, we have

$$
\rho_{3}^{1}(G) \geq \operatorname{la}(G) \geq \chi^{\prime}(G) / 2 \geq \Delta(G) / 2,
$$

where $\chi^{\prime}(G)$ denotes the chromatic index ${ }^{2}$ of $G$. On the other hand, $\rho_{3}^{1}(G)$ cannot be bounded from above in terms of la $(G)$. Indeed, let $G$ be an arbitrary cubic graph. Akiyama et al. [2] showed that $\mathrm{la}(G)=2$. On the other hand, any vertex of $G$ is essential, thus we have $\rho_{3}^{1}(G)>\sqrt{2 n}$ by Lemma $2.7($ a). Theorem 2.9 below shows an even larger gap.

Theorem 2.9. The following lower bound holds for almost all cubic graphs $G$ with $n$ vertices: $\rho_{3}^{1}(G)>n / 5$.

Proof. The bisection width $\mathrm{bw}(G)$ of a graph $G$ is the minimum possible number of edges between two sets of vertices $W_{1}$ and $W_{2}$ with $\left|W_{1}\right|=\lceil n / 2\rceil$ and $\left|W_{2}\right|=\lfloor n / 2\rfloor$ partitioning $V(G)$. We prove that $\operatorname{bw}(G) \leq \rho_{3}^{1}(G)$ for any graph $G$. Then the theorem follows from the fact that a random cubic graph on $n$ vertices has bisection width at least $n / 4.95$ with probability $1-o(1)$, which was shown by Kostochka and Melnikov [59].

Fix a drawing of the graph $G$ so that its edges lie on $r=\rho_{3}^{1}(G)$ lines in $\mathbb{R}^{3}$. Choose a plane $L$ that is not parallel to any of the at most $\left(\begin{array}{l}n \\ 2\end{array}\right)$ lines passing through two vertices of the drawing. Let us move $L$ along the orthogonal direction until it separates the vertex set of $G$ into two almost equal parts $W_{1}$ and $W_{2}$ (that is, ||$W_{1}|-| W_{2}|| \leq 1$ and no vertex of the graph $G$ belongs to $L$ ). The plane $L$ can intersect at most $r$ edges of $G$, which implies that $\operatorname{bw}(G) \leq r$.

Theorem 2.9 shows that graphs of bounded vertex degree can have linearly large value of $\rho_{3}^{1}(G)$. Hence, the factor of $n$ in the trivial bound $\rho_{3}^{1}(G) \leq m \leq \frac{1}{2} n \Delta(G)$ is best possible.

\footnotetext{
${ }^{2}$ also called the edge chromatic number
} 
We now prove a general lower bound for $\rho_{3}^{1}(G)$ in terms of the treewidth of $G$. The relationship between $\rho_{3}^{1}(G)$ and $\operatorname{tw}(G)$ follows from the fact that graphs with low parameter $\rho_{3}^{1}(G)$ have small separators, which is interesting by itself. We need the following definition. Let $W \subseteq V(G)$. A set of vertices $S \subset V(G)$ is a balanced $W$-separator of the graph $G$ if $|W \cap C| \leq|W| / 2$ for every connected component $C$ of $G \backslash S$. Let $\operatorname{sep}_{W}(G)$ denote the minimum $k$ such that $G$ has a balanced $W$-separator $S$ with $|S|=k$. It is known [44, Theorem 11.17] that $\operatorname{sep}_{W}(G) \leq \operatorname{tw}(G)+1$ for every $W \subseteq V(G)$. On the other hand, if $\operatorname{sep}_{W}(G) \leq k$ for all $W \subseteq V(G)$ with $|W|=2 k+1$, then $\operatorname{tw}(G) \leq 3 k$.

Lemma 2.10. For any graph $G, \rho_{3}^{1}(G) \geq \operatorname{sep}_{W}(G)$ for every $W \subseteq V(G)$.

Proof. Given $W \subseteq V(G)$, we have to prove that $\operatorname{sep}_{W}(G) \leq \rho_{3}^{1}(G)$. Fix a drawing of the graph $G$ so that its edges lie on $r=\rho_{3}^{1}(G)$ lines in $\mathbb{R}^{3}$, choose a plane $L$ as in the proof of Theorem 2.9 and move it until it separates $W$ into two equal parts $W_{1}$ and $W_{2}$. If $|W|$ is odd, then $L$ contains one vertex $w$ of $W$ and no other vertices of $G$. If $|W|$ is even, we can ensure that $L$ does not contain any vertex of $G$.

We now construct a set $S$ as follows. If $L$ contains a vertex $w \in W$, that is, $|W|$ is odd, we put $w$ in $S$. Let $E$ be the set of those edges which are intersected by $L$ but are not incident to the vertex $w$ (if it exists). Note that $|E|<r$ if $|W|$ is odd and $|E| \leq r$ if $|W|$ is even. Each of the edges in $E$ contributes one of its incident vertices into $S$. Note that $|S| \leq r$.

The plane $L$ splits the set of vertices $V(G) \backslash\{w\}$ into two parts, $V_{1} \supseteq W_{1}$ and $V_{2} \supseteq W_{2}$. The construction of $S$ ensures that there is no path between $V_{1}$ and $V_{2}$ avoiding $S$. Therefore, any connected component $C$ of $G \backslash S$ is included either in $V_{1}$ or in $V_{2}$. Thus, $C$ is disjoint from $W_{2} \cup\{w\}$ or from $W_{1} \cup\{w\}$, which implies that $|W \cap C| \leq|W| / 2$. We conclude that $S$ is a balanced $W$-separator.

The next theorem follows from Lemma 2.10 by the relationship between treewidth and balanced separators.

Theorem 2.11. For any graph $G, \rho_{3}^{1}(G) \geq \operatorname{tw}(G) / 3$.

On the other hand, $\rho_{3}^{1}(G)$ cannot be bounded from above by any function of $\operatorname{tw}(G)$, even for graphs of bounded degree. Indeed, by Lemma 2.7 (a) we have $\rho_{3}^{1}(T)=\Omega(\sqrt{n})$ for every caterpillar $T$ with linearly many vertices of degree 3 . The asymptotically best possible relation in this direction is $\rho_{3}^{1}(G) \leq m \leq n \operatorname{tw}(G)$; the second inequality is due to Chandran and Subramanian [16]. Note for comparison that $\pi_{3}^{1}(G) \leq \chi(G) \leq \operatorname{tw}(G)+1$. The factor of $n$ in the upper bound for $\rho_{3}^{1}(G)$ cannot be much improved, as follows from the next example.

Example 2.12. $\rho_{3}^{1}\left(K_{n}\right)=\left(\begin{array}{l}n \\ 2\end{array}\right)$ for any $n \geq 2$.

Proof. Any line contains at most one of the $\left(\begin{array}{l}n \\ 2\end{array}\right)$ edges of $K_{n}$, otherwise the line would contain a triangle. 


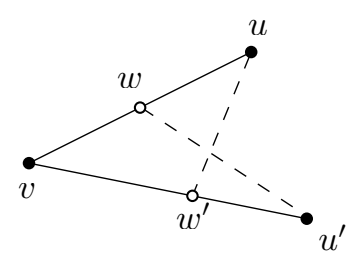

(a) $\operatorname{deg}_{H} v \leq 1$

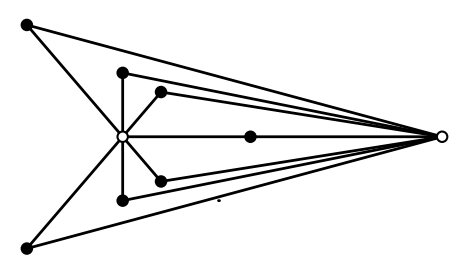

(b) $\rho_{2}^{1}\left(K_{2, q}\right) \leq 2 q-\lfloor(q+1) / 2\rfloor$

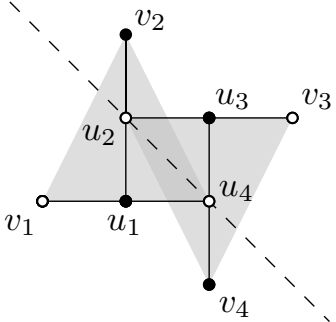

(c) $\rho_{3}^{1}\left(K_{4,4}\right) \leq 12$. The triangle $u_{2} v_{3} v_{4}$ is orthogonal to the triangle $v_{1} v_{2} u_{4}$; they intersect along the dashed line $u_{2} u_{4}$.

Figure 4. Establishing lower and upper bounds for $\rho_{*}^{1}\left(K_{p, q}\right)$ (proof of Example 2.13).

We are also able to determine the exact values of $\rho_{2}^{1}(G)$ and $\rho_{3}^{1}(G)$ for complete bipartite graphs $K_{p, q}$.

Example 2.13. The line cover numbers of the complete bipartite graphs are as follows.

(a) For $q \geq 1, \rho_{3}^{1}\left(K_{1, q}\right)=\rho_{2}^{1}\left(K_{1, q}\right)=q-\lfloor q / 2\rfloor=\lceil m / 2\rceil$.

(b) For $q \geq 2, \rho_{3}^{1}\left(K_{2, q}\right)=\rho_{2}^{1}\left(K_{2, q}\right)=2 q-\lfloor(q+1) / 2\rfloor=\lceil(3 m-2) / 4\rceil$.

(c) For $q \geq p \geq 3, \rho_{3}^{1}\left(K_{p, q}\right)=p q-\lfloor p / 2\rfloor-\lfloor q / 2\rfloor$.

Proof. Since (a) is obvious, we only consider (b) and (c). Suppose that the bipartition of the graph $K_{p, q}$ is defined by $p$ white and $q$ black vertices.

To show the lower bound (for both cases), fix an optimal set $\mathcal{L}$ of lines covering the edges of $K_{p, q}$ and consider an auxiliary graph $H$ on the same vertex set as the graph $K_{p, q}$ and the set of edges defined as follows. Each line $\ell \in \mathcal{L}$ contains two vertices of $K_{p, q}$ with different colors or two monochromatic vertices $v$ and $u$ of $K_{p, q}$ and a vertex $w$ of $K_{p, q}$ with another color, which is drawn inside the segment $[v u]$ between the vertices $v$ and $u$. In the latter case, we shall consider the vertices $v$ and $u$ of the graph $H$ as adjacent. We claim that each vertex $v$ of the graph $H$ has degree at most one. Indeed, assume the converse, the vertex $v$ is adjacent to distinct vertices $u$ and $u^{\prime}$. Then there exist (necessarily distinct) vertices $w$ and $w^{\prime}$ drawn inside the segments $[v u]$ and $\left[v u^{\prime}\right]$, respectively. This implies that the segments $\left[w u^{\prime}\right]$ and $\left[w^{\prime} u\right]$ intersect (see Fig. 4a), which is impossible, a contradiction. Thus, the number $m_{H}$ of edges of the graph $H$ is at most $l(p, q)=\lfloor p / 2\rfloor+\lfloor q / 2\rfloor$. Since the number $m$ of edges of the graph $K_{p, q}$ equals $p q$ and $|\mathcal{L}|=m-m_{H} \geq p q-\lfloor p / 2\rfloor-\lfloor q / 2\rfloor$, we already obtain a lower bound for Case (c). For Case (b) we shall be a bit more specific and take care of the case of even $q$. It suffices to show that $m_{H} \leq l(p, q)-1$. If the two white vertices $v$ and $u$ of the graph $K_{2, q}$ are not adjacent in the graph $H$, then $m_{H} \leq q / 2=l(p, q)-1$. Assume that the vertices $v$ and $u$ are adjacent in $H$. Then there exists a black vertex $w$ of the graph $K_{2, q}$ that lies inside the segment $[v u]$. It follows that the vertex $w$ cannot be adjacent to any other vertex in $H$. Therefore, $m_{H} \leq 1+q / 2-1=l(p, q)-1$.

For Case (b), the upper bound $\rho_{3}^{1}\left(K_{2, q}\right) \leq \rho_{2}^{1}\left(K_{2, q}\right) \leq 2 q-\lfloor(q+1) / 2\rfloor$ is shown in Fig. 4b. To show the upper bound for Case (c), it suffices to present drawings of the graph $K_{p, q}$ which certify the equality $m_{H}=l(p, q)$. We prove this by induction. For the 
base case, we describe drawings of the graphs $K_{3,3}, K_{3,4}$, and $K_{4,4}$. For the last graph in this list, a partial drawing is shown in Fig. 4c. The corresponding auxiliary graph $H$ consists of the following edges: $\left\{v_{1}, u_{4}\right\},\left\{v_{2}, u_{1}\right\},\left\{v_{3}, u_{2}\right\}$, and $\left\{v_{4}, u_{3}\right\}$. This shows $m_{H}=4=l(4,4)$. The drawing of the graph $K_{3,4}$ can be obtained from the drawing of the graph $K_{4,4}$ by removing its vertex $v_{1}$; the drawing of $K_{3,3}$ can be obtained by removing $v_{1}$ and $v_{2}$.

For the inductive step, observe that if a drawing of $K_{p, q}$ satisfying $m_{H}=l(p, q)$ exists for a pair $(p, q)$, then drawings with $m_{H}=l(p+2, q)$ and with $m_{H}=l(p, q+2)$ also exist for the pairs $(p+2, q)$ and $(p, q+2)$, respectively. To see this, we can add a pair of vertices $u$ and $v$ of the same color to the graph $K_{p, q}$ such that the segment [uv] contains a vertex of the graph $G$ of the other color. We can assure the absence of crossings in the new graph if both vertices $u$ and $v$ will be taken outside any plane spanned by three distinct vertices of the graph $K_{p, q}$. Similarly, if $p$ is even, then such a drawing also exists for a pair $(p+1, q)$ because for this purpose we can add a white vertex to the graph $K_{p, q}$ without creating new edges in the corresponding graph $H$. Correspondingly, if $q$ is even, then such a drawing also exists for a pair $(p, q+1)$.

\subsection{Placing Edges on Few Planes $\left(\rho_{3}^{2}\right)$}

We now turn to the plane cover number. At the end of this section, we will establish lower and upper bounds for the plane cover number of complete graphs and determine exact values for complete graphs with up to 9 vertices.

But the first result is about almost-planar graphs, that is, graphs that become planar after the deletion of a single edge. If we use more than one plane, we can avoid crossings. By lifting one of the endpoints of the additional edge above the $x y$-plane, it is easy to upper-bound the number of planes in terms of the degree of this endpoint. We show that a constant number of planes suffices. For two vertices $u$ and $v$ of a graph $G$, let $G+u v$ denote the graph obtained from $G$ by connecting $u$ and $v$ by an edge (if they were not adjacent).

Theorem 2.14. For any planar graph $G$ and any pair of vertices $u$ and $v$ of $G$, it holds that $\rho_{3}^{2}(G+u v) \leq 4$.

Proof. We first triangulate $G$ by adding edges without introducing crossings. If this makes $u$ and $v$ adjacent, we are done since then $G+u v$ is planar. Otherwise, take a Schnyder realizer $\left(\mathcal{T}_{1}, \mathcal{T}_{2}, \mathcal{T}_{3}\right)$ of the resulting triangulation $G^{\prime}[76]$. Each of the three trees in the realizer spans the interior vertices of $G^{\prime}$ and is rooted at a distinct vertex of the outer face. We direct the trees towards their roots. We say that an edge that belongs to tree $\mathcal{T}_{i}$ has color $i$. In each interior vertex, the three outgoing edges (each belonging to a distinct tree) have the same cyclic order. In this cyclic order, the incoming edges of one tree all lie between the two outgoing edges of the two other trees. The three trees connect each interior vertex $w$ by paths $\mathcal{P}_{1}(w), \mathcal{P}_{2}(w)$, and $\mathcal{P}_{3}(w)$ to the exterior vertices $a_{1}, a_{2}$, and $a_{3}$, respectively. The paths partition the triangulation into three regions $\mathcal{R}_{1}(w), \mathcal{R}_{2}(w)$, and $\mathcal{R}_{3}(w)$ with respect to $w$; region $\mathcal{R}_{1}(w)$ is bounded by paths $\mathcal{P}_{2}(w)$ and $\mathcal{P}_{3}(w)$ and so on.

Due to the cyclic order of the in- and outgoing edges around each vertex $w$, it is clear that the three paths $\mathcal{P}_{1}(w), \mathcal{P}_{2}(w)$, and $\mathcal{P}_{3}(w)$ only meet in the starting point. This 


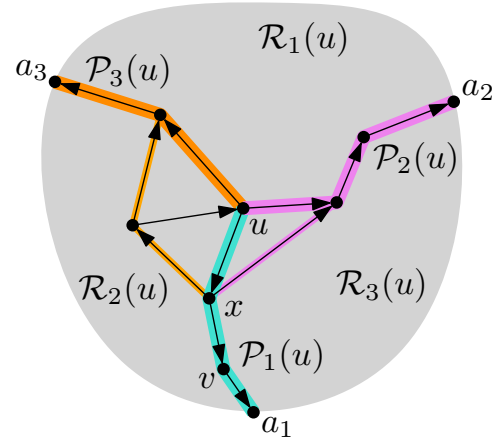

(a) case I (paths from $u$ bold)

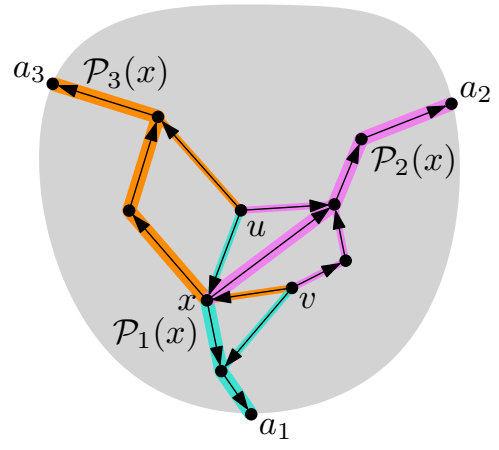

(b) case II (paths from $x$ bold)

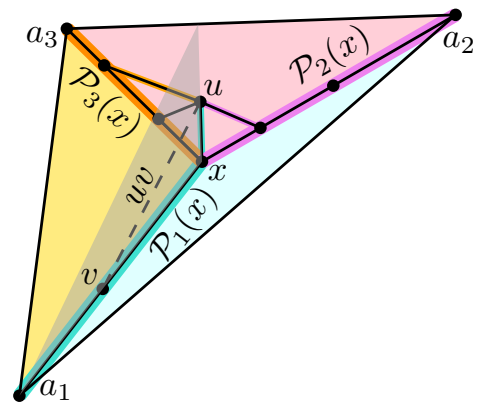

(c) drawing on four planes (case I)

Figure 5. Illustration for the proof of Theorem 2.14.

in turn shows that paths $\mathcal{P}_{i}(w)$ and $\mathcal{P}_{i^{\prime}}\left(w^{\prime}\right)$ for two vertices $w \neq w^{\prime}$ and indices $i \neq i^{\prime}$ meet at most once. This implies that none of these paths can have a chord, which is important for our construction.

We want to find a vertex $x \notin\{u, v\}$ that "separates" $u$ and $v$ in the sense that $u$ and $v$ end up in different regions with respect to $x$ and that $u$ and $v$ do not both belong to $\mathcal{P}_{i}(x)$ for any $i \in\{1,2,3\}$. Then we can draw $G$ on three faces of a tetrahedron with $x$ on the tip, paths $\mathcal{P}_{1}(x), \mathcal{P}_{2}(x)$, and $\mathcal{P}_{3}(x)$ on the edges incident to the tip, and edge $u v$ as a line segment in the interior of the tetrahedron. Consider the paths $\mathcal{P}_{i}(u)$ and $\mathcal{P}_{i}(v)$ that connect $u$ and $v$ to the exterior vertices. We distinguish two cases depending on whether or not one of the vertices $u$ and $v$ lies on one of the paths emanating from the other vertex.

Case I: See Fig. 5a. Vertex $v$ lies on $\mathcal{P}_{i}(u)$ or $u$ lies on $\mathcal{P}_{i}(v)$ for some $i \in\{1,2,3\}$. Without loss of generality, assume that $v$ lies on $\mathcal{P}_{1}(u)$. In this case, let $x$ be the successor of $u$ on $\mathcal{P}_{1}(u)$. As $x$ is a neighbor of $u$ but $u v$ is not an edge of $G^{\prime}$, we know that $x \neq v$. Also note that, for $j \in\{2,3\},\{u, v\} \cap \mathcal{P}_{j}(x)=\emptyset$ due to the cyclic order of the edges around the vertices.

Case II: See Fig. 5b. For each tree $\mathcal{T}_{i}$, there are two paths from the root to $u$ and $v$, respectively. These paths may share edges at the beginning, but none of them is a subset of the other (otherwise, we would be in case I). Without loss of generality, we assume that $v$ lies in the region $\mathcal{R}_{3}(u)$ and that the path $\mathcal{P}_{3}(v)$ crosses $\mathcal{P}_{1}(u)$; the other cases are symmetric. Let $x$ be the crossing vertex of $\mathcal{P}_{3}(v)$ and $\mathcal{P}_{1}(u)$. Note that $x \notin\{u, v\}$ given our assumption that, for $i \in\{1,2,3\}$, neither $\mathcal{P}_{i}(u)$ is a subpath of $\mathcal{P}_{i}(v)$ nor the other way around. As $x \in \mathcal{P}_{3}(v), v$ lies in (the interior of) $\mathcal{R}_{3}(x)$ by the construction of the Schnyder realizer. Analogously, as $x \in \mathcal{P}_{1}(u), u$ lies in (the interior of) $\mathcal{R}_{1}(x)$.

Hence, in both cases, $x$ separates $u$ and $v$ as desired.

We draw $G^{\prime}$ into an equilateral triangle $T$. The exterior vertices of $G^{\prime}$ are mapped to the vertices of the triangle, $x$ is mapped to the centroid of $T$, and the three paths $\mathcal{P}_{1}(x)$, 
$\mathcal{P}_{2}(x)$, and $\mathcal{P}_{3}(x)$ are mapped to straight-line segments that connect $x$ to the exterior vertices. This is possible since the paths do not have chords. The paths subdivide $T$ into three congruent isosceles triangles $T_{1}, T_{2}$, and $T_{3}$. We use Tutte's barycenter algorithm [81] three times, to draw each region $\mathcal{R}_{i}$ into the corresponding triangle $T_{i}$. Then we project this planar drawing onto three faces of a tetrahedron as described above. Finally, as a fourth plane, we add any plane that contains $u$ and $v$. In Fig. $5 \mathrm{c}$, we chose the gray plane that additionally contains $x$.

Problem 1. Can we bound $\rho_{3}^{2}$ (or $\pi_{3}^{2}$ ) for the class of 1-planar graphs (which are the graphs that can be drawn in $\mathbb{R}^{2}$ with at most one crossing per edge) or for the class of RAC graphs (which are the graphs that can be drawn straight-line in $\mathbb{R}^{2}$ such that all crossings are at right angles)?

Example 2.15. For any integers $1 \leq p \leq q$, it holds that $\rho_{3}^{2}\left(K_{p, q}\right)=\lceil p / 2\rceil$.

Proof. If $p \leq 2$, the graph $K_{p, q}$ is planar, and the equality reads $\rho_{3}^{2}\left(K_{p, q}\right)=1$. Suppose that $p \geq 3$. Let $\mathcal{L}$ be a set of planes covering a drawing of $K_{p, q}$. Every plane in $\mathcal{L}$ covers at most $2 q$ edges, for else it would contain the non-planar $K_{3,3}$. Since the total number of edges is $p q$, we conclude that $|\mathcal{L}| \geq\lceil p q / 2 q\rceil=\lceil p / 2\rceil$.

A drawing showing tightness of this lower bound is similar to a standard book embedding of $K_{p, q}$ on $p$ pages. Denote the vertex classes of $K_{p, q}$ by $V_{1}$ and $V_{2}$ where $\left|V_{1}\right|=p$ and $\left|V_{2}\right|=q$. Place all the vertices in $V_{2}$ on a line $\ell$ and consider $\lceil p / 2\rceil$ distinct planes containing $\ell$. The line $\ell$ divides the planes into $2\lceil p / 2\rceil \geq p$ half-planes. Put every vertex in $V_{1}$ on one of these half-planes and connect it to the points on $\ell$.

Determining the parameter $\rho_{3}^{2}(G)$ for complete graphs $G=K_{n}$ is a much more subtle issue. We are able to determine the asymptotics of $\rho_{3}^{2}\left(K_{n}\right)$ up to a factor of 2 .

By a combinatorial cover of a graph $G$, we mean a set of subgraphs of $G$ such that every edge of $G$ belongs to at least one of these subgraphs. A geometric cover of a crossingfree drawing $d: V(G) \rightarrow \mathbb{R}^{3}$ of a graph $G$ is a set $\mathcal{L}$ of planes in $\mathbb{R}^{3}$ such that every edge in the drawing is contained in one of these planes. Any geometric cover $\mathcal{L}$ induces a combinatorial cover $\mathcal{K}_{\mathcal{L}}=\left\{G_{\ell}: \ell \in \mathcal{L}\right\}$ of $G$ where $G_{\ell}$ is the subgraph of $G$ formed by the edges drawn in the plane $\ell$. Note that, if $G=K_{n}$, then each $G_{\ell}$ is a $K_{s}$ subgraph with $s \leq 4$ (because $K_{5}$ is not planar).

Given $k \leq n$, let $c\left(K_{n}, K_{k}\right)$ denote the minimum size of a combinatorial cover of $K_{n}$ by $K_{k}$ subgraphs. Obviously, $c\left(K_{n}, K_{k}\right) \geq \frac{n(n-1)}{k(k-1)}$. This lower bound is attained if and only if there exists a Steiner system $S(2, k, n)$. Note that, in our terminology, a Steiner system $S(2, k, n)$ is a combinatorial cover of $K_{n}$ by $K_{k}$ subgraphs such that every edge of $K_{n}$ belongs to exactly one of these subgraphs. In 1847 Kirkman showed (see, for example, Bollobás' book [10, page 113]) that a Steiner system $S(2,3, n)$ exists if and only if $n \equiv 1(\bmod 6)$ or $n \equiv 3(\bmod 6)$. For example, Fig. 6 b shows that $c\left(K_{7}, K_{3}\right)=7$. We get an upper bound for the size of a combinatorial cover by adding vertices to the graph until a Steiner system exists for $n$. It follows that $\left(n^{2}-n\right) / 6 \leq c\left(K_{n}, K_{3}\right) \leq(n+3)(n+2) / 6=\left(n^{2}+5 n+6\right) / 6$ for any $n \geq 3$. More than one hundred years later, Hanani [50] (see also [73, Theorem 2.1]) 


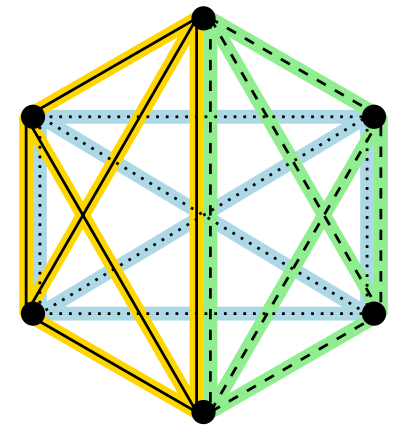

(a) $c\left(K_{6}, K_{4}\right)=3$.

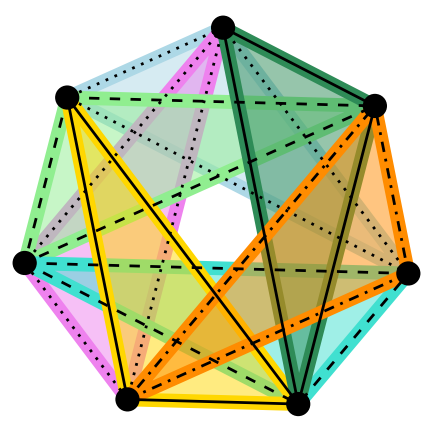

(b) $c\left(K_{7}, K_{3}\right)=7$.

Figure 6. Combinatorial covers of $K_{6}$ and $K_{7}$ with $K_{4}$ and $K_{3}$, respectively.

showed that a Steiner system $S(2,4, n)$ exists if and only if $n \equiv 1(\bmod 12)$ or $n \equiv 4(\bmod 12)$. It follows that we have

$$
\left(n^{2}-n\right) / 12 \leq c\left(K_{n}, K_{4}\right) \leq(n+8)(n+7) / 12=\left(n^{2}+15 n+56\right) / 12
$$

for any $n \geq 4$. This leads to the following bounds for $\rho_{3}^{2}\left(K_{n}\right)$.

Theorem 2.16. For all $n \geq 4$,

$$
\frac{n^{2}-n}{12} \leq c\left(K_{n}, K_{4}\right) \leq \rho_{3}^{2}\left(K_{n}\right) \leq c\left(K_{n}, K_{3}\right) \leq \frac{n^{2}+5 n+6}{6} .
$$

Proof. For the lower bound, consider a drawing of $K_{n}$ that admits a geometric cover $\mathcal{L}$ by $\rho_{3}^{2}\left(K_{n}\right)$ planes. This geometric cover induces a combinatorial cover $\mathcal{K}_{\mathcal{L}}$ of $K_{n}$ by $\rho_{3}^{2}\left(K_{n}\right)$ copies of $K_{4}, K_{3}$, or $K_{2}$. It follows that $c\left(K_{n}, K_{4}\right) \leq \rho_{3}^{2}\left(K_{n}\right)$.

For the upper bound, let $d: V\left(K_{n}\right) \rightarrow \mathbb{R}^{3}$ be an arbitrary crossing-free straightline drawing of $K_{n}$ in 3-space. Let $\mathcal{K}$ be a combinatorial cover of $K_{n}$ by $c\left(K_{n}, K_{3}\right) K_{3^{-}}$ subgraphs. For each subgraph $K \in \mathcal{K}$, let $\ell_{K}$ be the plane containing the $d$-image of $K$. Then $\left\{\ell_{K}: K \in \mathcal{K}\right\}$ is a geometric cover of $d$ certifying that $\rho_{3}^{2}\left(K_{n}\right) \leq c\left(K_{n}, K_{3}\right)$.

Problem 2. Can the gap between the bounds for $\rho_{3}^{2}\left(K_{n}\right)$ in Theorem 2.16 be made closer? Find $\lim _{n \rightarrow \infty} \rho_{3}^{2}\left(K_{n}\right) / n^{2}$ if it exists.

Note that we cannot always realize a combinatorial cover of $K_{n}$ by copies of $K_{4}$ geometrically. For example, Fig. 6 a shows that $c\left(K_{6}, K_{4}\right)=3$ while $\rho_{3}^{2}\left(K_{6}\right)=4$ by Theorem 2.19 below.

In order to determine $\rho_{3}^{2}\left(K_{n}\right)$ for particular values of $n$, we need some properties of geometric and combinatorial covers of $K_{n}$.

Lemma 2.17. If $d: V\left(K_{n}\right) \rightarrow \mathbb{R}^{3}$ is a crossing-free drawing of $K_{n}$ and $\mathcal{L}$ is a geometric cover of $d$, then two different 4 -vertex subgraphs $G_{\ell}$ and $G_{\ell^{\prime}}$ in $\mathcal{K}_{\mathcal{L}}$ can share at most two vertices. 
Table 1. Lower and upper bounds for $\rho_{3}^{2}\left(K_{n}\right)$ for small values of $n$.

\begin{tabular}{rrrrrrr}
\hline$n$ & 4 & 5 & 6 & 7 & 8 & 9 \\
\hline$\geq$ & 1 & 3 & 4 & 6 & 6 & 7 \\
$\leq$ & 1 & 3 & 4 & 6 & 7 & 10 \\
\hline
\end{tabular}

Proof. Assume, to the contrary, that $G_{\ell}$ and $G_{\ell^{\prime}}$ share three vertices. Since these vertices cannot be collinear, we have $\ell=\ell^{\prime}$. This implies that $G_{\ell}=G_{\ell^{\prime}}$, yielding a contradiction.

Lemma 2.18. The following statements hold for all $n \geq 4$.

(a) $\rho_{3}^{2}\left(K_{n+1}\right) \leq \rho_{3}^{2}\left(K_{n}\right)+\lceil n / 2\rceil$.

(b) $\rho_{3}^{2}\left(K_{n+1}\right) \leq \rho_{3}^{2}\left(K_{n}\right)+\lceil(n-3) / 2\rceil$ if there is a geometric cover of a drawing d of $K_{n}$ by $\rho_{3}^{2}\left(K_{n}\right)$ planes such that one of the covering planes contains exactly three vertices.

Proof. Let $V\left(K_{n}\right)=\left\{v_{1}, \ldots, v_{n}\right\}$ and $V\left(K_{n+1}\right)=V\left(K_{n}\right) \cup\left\{v_{n+1}\right\}$.

(a) Any drawing $d$ of $K_{n}$ can be extended to a crossing-free drawing of $K_{n+1}$ by adding a new point $d\left(v_{n+1}\right)$ and connecting it by segments to $d\left(v_{i}\right)$ for all $i \leq n$. The new $n$ segments can be covered by $\lceil n / 2\rceil$ planes.

(b) Without loss of generality, suppose that a covering plane $\ell$ contains exactly three vertices $d\left(v_{1}\right), d\left(v_{2}\right)$, and $d\left(v_{3}\right)$. We can extend $d$ to a crossing-free drawing of $K_{n+1}$ by adding a new point $d\left(v_{n+1}\right)$ inside of the triangle $d\left(v_{1}\right) d\left(v_{2}\right) d\left(v_{3}\right)$. The plane $\ell$ covers the segments $d\left(v_{n+1}\right) d\left(v_{i}\right)$ for $i \leq 3$, and $\lceil(n-3) / 2\rceil$ new planes suffice to cover the segments $d\left(v_{n+1}\right) d\left(v_{i}\right)$ for $3<i \leq n$.

Theorem 2.19. For $n \leq 9$, the value of $\rho_{3}^{2}\left(K_{n}\right)$ is bounded by the numbers in Table 1.

Proof. Case $n=5$ : By Lemma 2.18(a), $\rho_{3}^{2}\left(K_{5}\right) \leq \rho_{3}^{2}\left(K_{4}\right)+2=3$. This bound is tight because $\rho_{3}^{2}\left(K_{5}\right) \geq c\left(K_{5}, K_{4}\right) \geq 3$. To prove the last inequality, note that although a graph $K_{5}$ has $10<2 \cdot 6$ edges, it cannot be covered by two $K_{4}$ subgraphs $K_{4}^{\prime}$ and $K_{4}^{\prime \prime}$. Indeed, in this case $K_{4}^{\prime}$ and $K_{4}^{\prime \prime}$ would have at least three common vertices and, hence, at least three common edges, whereas $12-3<10$.

Case $n=6$ : Fig. 1 shows that $\rho_{3}^{2}\left(K_{6}\right) \leq 4$. Now we argue that $\rho_{3}^{2}\left(K_{6}\right) \geq 4$. Assume, to the contrary, that $K_{6}$ has a drawing $d$ with a geometric cover $\mathcal{L}$ by 3 planes. Consider the corresponding combinatorial cover $\mathcal{K}_{\mathcal{L}}$ of $K_{6}$. Since $K_{6}$ has 15 edges, $\mathcal{K}_{\mathcal{L}}$ has to use at least two copies of $K_{4}$ and, additionally, a copy of $K_{k}$ for $3 \leq k \leq 4$. Note now that any two $K_{4}$ subgraphs of $K_{6}$ have a common edge and, by Lemma 2.17, this edge is unique. It follows from here that the cover $\mathcal{K}_{\mathcal{L}}$ consists of three $K_{4}$ subgraphs. Denote them by $K_{4}^{1}, K_{4}^{2}$, and $K_{4}^{3}$. Since $K_{4}$ is not outerplanar, each $K_{4}^{i}$ is represented in $d$ by a triangle with an additional point $d\left(v_{i}\right)$ in its interior, $i=1,2,3$. Let $V_{0}=\left\{v_{1}, v_{2}, v_{3}\right\}$. By the Krein-Milman theorem [60,84], the convex hull $\operatorname{Conv}\left(d\left(V\left(K_{6}\right)\right)\right)$ coincides with the convex hull $\operatorname{Conv}\left(d\left(V\left(K_{6}\right)\right) \backslash d\left(V_{0}\right)\right)$. If all the vertices $v_{i}$ are mutually distinct, then the set $d\left(V\left(K_{6}\right)\right) \backslash d\left(V_{0}\right)$ must be a triangle and the drawing $d$ is contained in a single 
plane, a contradiction. Therefore, $v_{i}=v_{j}$ for some $i \neq j$. Let $k$ be the third index that is distinct from both $i$ and $j$. The unique common edge of $K_{4}^{i}$ and $K_{4}^{j}$ has to connect the vertex $v_{i}$ to some other vertex $v$ of $K_{6}$ (see Fig. 1 with $u_{4}$ for $v_{i}$ and $u_{1}$ for $v$ ). Let $V\left(K_{4}^{i}\right)=\left\{v, v_{i}, v_{i}^{1}, v_{i}^{2}\right\}$ and $V\left(K_{4}^{j}\right)=\left\{v, v_{j}, v_{j}^{1}, v_{j}^{2}\right\}$. Since $K_{4}^{1}, K_{4}^{2}$, and $K_{4}^{3}$ cover all edges of $K_{6}$, the edges $v_{i}^{1} v_{j}^{1}, v_{i}^{1} v_{j}^{2}, v_{i}^{2} v_{j}^{1}$, and $v_{i}^{2} v_{j}^{2}$ all belong to $K_{4}^{k}$. Therefore, we have $V\left(K_{4}^{k}\right)=\left\{v_{i}^{1}, v_{i}^{2}, v_{j}^{1}, v_{j}^{2}\right\}$. However, vertices $v_{i}^{1}, v_{i}^{2}, v_{j}^{1}$, and $v_{j}^{2}$ are in convex position (see Fig. 1), contradicting the fact that $K_{4}$ is not outerplanar.

Case $n=7$ : Fig. 1 shows the cover of a drawing of $K_{6}$ by four planes, and one of them contains exactly three vertices. By Lemma 2.18(b), we have $\rho_{3}^{2}\left(K_{7}\right) \leq 4+\lceil(6-3) / 2\rceil=6$. Now we argue that $\rho_{3}^{2}\left(K_{7}\right) \geq 6$. Assume, to the contrary, that $K_{7}$ has a drawing with a geometric cover $\mathcal{L}$ by $r<6$ planes. Consider the corresponding combinatorial cover $\mathcal{K}_{\mathcal{L}}$ of $K_{7}$. Let $s$ denote the number of pairs $(u, K)$ such that $u \in V(K)$ and $K \in \mathcal{K}_{\mathcal{L}}$. Since $\left|\mathcal{K}_{\mathcal{L}}\right|=r$ and each subgraph $K \in \mathcal{K}_{\mathcal{L}}$ has at most four vertices, $s \leq 5 \cdot 4=20<21=7 \cdot 3$. Therefore, there exists a vertex $v \in V\left(K_{7}\right)$ that belongs to at most two subgraphs in $\mathcal{K}_{\mathcal{L}}$. Since each $K \in \mathcal{K}_{\mathcal{L}}$ contains at most three edges incident to $v$ (and exactly three only if $K$ is a $K_{4}$ subgraph containing $v$ ) and $v$ is incident to six edges of $K_{7}$, we see that $v$ belongs to exactly two subgraphs $K_{4}^{1}$ and $K_{4}^{2}$ in $\mathcal{K}_{\mathcal{L}}$ and each of them is a copy of $K_{4}$. Moreover, $v$ is the only common vertex of $K_{4}^{1}$ and $K_{4}^{2}$. For each $i=1,2$, let $V_{i}=V\left(K_{4}^{i}\right) \backslash\{v\}$. Let $\mathcal{K}^{\prime}=\mathcal{K}_{\mathcal{L}} \backslash\left\{K_{4}^{1}, K_{4}^{2}\right\}$. Since $\left|\mathcal{K}^{\prime}\right|=r-2$ and $r-2<4=\rho_{3}^{2}\left(K_{6}\right)$, the graph $K_{7}-v$ contains an edge - call it $v^{\prime} v^{\prime \prime}$ - that does not belong to any subgraph in $\mathcal{K}^{\prime}$. Since every edge between $V_{1}$ and $V_{2}$ belongs to a subgraph in $\mathcal{K}^{\prime}$, there exists an index $i \in\{1,2\}$ such that $V^{\prime}=\left\{v^{\prime}, v^{\prime \prime}\right\} \subset V_{i}$. Let $j \neq i$ be the other index in $\{1,2\}$. We have $\left|V^{\prime} \cap V(K)\right| \leq 1$ for every $K \in \mathcal{K}^{\prime}$. Since $\left|\mathcal{K}^{\prime}\right|=r-2 \leq 3$, there exists a vertex $w^{\prime} \in V^{\prime}$ that belongs to at most one subgraph $K \in \mathcal{K}^{\prime}$. Such a subgraph $K$ does exist because otherwise no edge $w^{\prime} w$ with $w \in V_{j}$ would be covered by $\mathcal{K}_{\mathcal{L}}$. Since both $K_{4}^{j}$ and $K$ are members of $\mathcal{K}_{\mathcal{L}}$, Lemma 2.17 implies that there exists a vertex $w \in V_{j} \backslash V(K)$. However, the edge $w^{\prime} w$ is not covered by $\mathcal{K}_{\mathcal{L}}$, a contradiction.

Case $n=8$ : Clearly, $\rho_{3}^{2}\left(K_{8}\right) \geq \rho_{3}^{2}\left(K_{7}\right)=6$. Let $d$ be the drawing of $K_{6}$ with vertices $u_{1}, \ldots, u_{6}$ on four planes depicted in Fig. 1. Extend this drawing by putting vertex $u_{7}$ into the interior of the purple triangle $d\left(u_{2}\right) d\left(u_{5}\right) d\left(u_{6}\right)$ and vertex $u_{8}$ into the interior of the orange triangle $d\left(u_{3}\right) d\left(u_{5}\right) d\left(u_{6}\right)$, symmetrically with respect to the axis $d\left(u_{1}\right) d\left(u_{4}\right)$ and such that the new points are not contained in the plane spanned by the blue triangle $d\left(u_{1}\right) d\left(u_{2}\right) d\left(u_{3}\right)$. In order to cover the resulting drawing of $K_{8}$, consider the four planes of Fig. 1 and an additional plane spanned by the triangle $d\left(u_{1}\right) d\left(u_{7}\right) d\left(u_{8}\right)$, which contains $d\left(u_{4}\right)$ due to the symmetric placement of $u_{7}$ and $u_{8}$. Note that only the two new edges $u_{3} u_{7}$ and $u_{2} u_{8}$ remain uncovered. Therefore, $\rho_{3}^{2}\left(K_{8}\right) \leq 7$.

Case $n=9$ : To see the upper bound, consider the drawing of $K_{8}$ again. In the last step, use the plane spanned by the triangle $d\left(u_{3}\right) d\left(u_{6}\right) d\left(u_{7}\right)$ to cover the edge $d\left(u_{3}\right) d\left(u_{7}\right)$. So we can apply Lemma 2.18(b) to get the upper bound of 10. For the lower bound, it suffices to prove $c\left(K_{9}, K_{4}\right)>6$ as $\rho_{3}^{2}\left(K_{9}\right) \geq c\left(K_{9}, K_{4}\right)$. Since a graph $K_{9}$ has $36=6 \cdot 6$ edges, each cover of $K_{9}$ by six $K_{4}$ subgraphs forms a Steiner system $S(2,4,9)$. The non-existence of such a system follows from the aforementioned result by Hanani and also can be seen directly by the following argument. Assume, to the contrary, that $K_{9}$ admits a combinatorial cover $\mathcal{K}^{\prime}$ by six $K_{4}$ subgraphs. Having degree 8, each vertex of $K_{9}$ belongs to at least 3 
members of $\mathcal{K}^{\prime}$. Let $s$ denote the number of pairs $(u, K)$ such that $u \in V(K)$ and $K \in \mathcal{K}^{\prime}$. It follows that $s \geq 9 \cdot 3=27$. On the other hand, each member $K \in \mathcal{K}^{\prime}$ contains 4 vertices, implying $s \leq 6 \cdot 4=24$, a contradiction.

Problem 3. Is $\rho_{3}^{2}(G)$ bounded by a constant or by a linear function for bounded-degree (in particular, cubic) graphs?

\section{The Line Cover Numbers of Planar Graphs in 3-Space and in the Plane}

In this section we focus on the line cover numbers of planar graphs, both in 2D and 3D. We start by showing a constant upper bound for the weak line cover number in 3D, including specific results about certain subclasses of planar graphs. After that, we establish a superlogarithmic lower bound for the weak line cover number in 2D for certain graphs, which shows an advantage of 3D drawings for these graphs. Also, there are classes of planar graphs for which the weak line cover numbers in 2D have a constant upper bound.

In the last subsection we relate the line cover number to the slope number and segment number and give examples that show gaps between these parameters. Again, we construct a graph class that shows a separation of 2D and 3D line cover numbers. Finally, we consider the line cover numbers of complete bipartite graphs and complete binary trees.

\subsection{Placing Vertices on Few Lines in 3-Space $\left(\pi_{3}^{1}\right)$}

Call a drawing outerplanar if all the vertices lie on the outer face. An outerplanar graph is a graph admitting an outerplanar drawing. Note that this definition does not depend on whether straight line or curved drawings are considered.

Combining Corollary 2.2 with the 4 -color theorem yields $\pi_{3}^{1}(G) \leq 4$ for planar graphs. Given that outerplanar graphs are 3-colorable (they are partial 2-trees), we obtain $\pi_{3}^{1}(G) \leq 3$ for these graphs. These bounds can be improved using the equality $\pi_{3}^{1}(G)=\operatorname{lva}(G)$ of Theorem 2.1 and known results on the linear vertex arboricity:

(a) For any planar graph $G$, it holds that $\pi_{3}^{1}(G) \leq 3[47,69]$.

(b) There is a planar graph $G$ with $\pi_{3}^{1}(G)=3[19,47]$.

(c) For any outerplanar graph $G, \pi_{3}^{1}(G) \leq 2[1,12,83]$.

According to Chen and He [20], the upper bound lva $(G) \leq 3$ for planar graphs by Poh [69] is constructive and yields a polynomial-time algorithm for partitioning the vertex set of a given planar graph into three parts, each inducing a linear forest. By combining this with the construction given in Theorem 2.1, we obtain a polynomial-time algorithm that draws a given planar graph such that the vertex set "sits" on three lines.

The example of Chartrand and Kronk [19] is a 21-vertex planar graph whose vertex arboricity is 3, which means that the vertex set of this graph cannot even be split into two parts both inducing (not necessarily linear) forests. Raspaud and Wang [71] showed that all 20-vertex planar graphs have vertex arboricity at most 2 . We now observe that a 


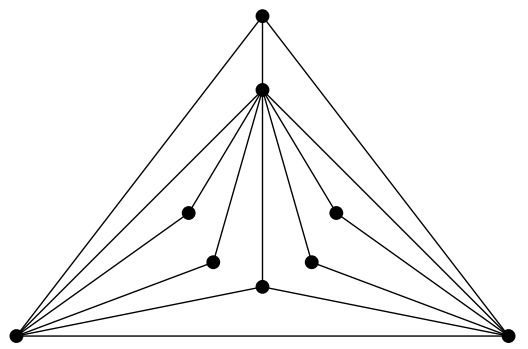

Figure 7. The smallest planar graph $G$ with $\operatorname{lva}(G)=3$.

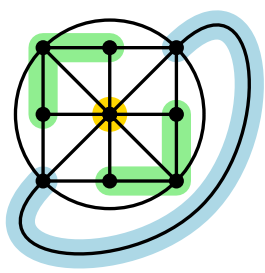

(a)

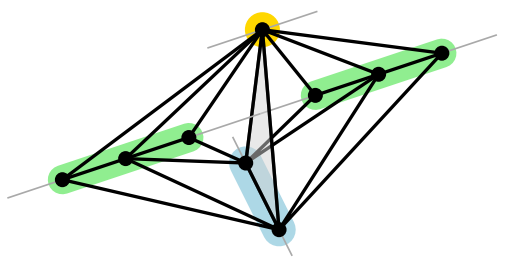

(b)
Figure 8. (a) Planar 9-vertex graph $G$ with $\pi_{3}^{1}(G)=3$ and (b) a $3 \mathrm{D}$ drawing of $G$ on three lines. (Copy of Fig. 2.)

smaller example of a planar graph attaining the extremal value $\pi_{3}^{1}(G)=3$ can be found by examining the linear vertex arboricity.

Example 3.1. The planar 9-vertex graph $G$ in Fig. 8 has $\pi_{3}^{1}(G)=\operatorname{lva}(G)=3$.

Proof. Indeed, in the picture it is easy to see that $2 \leq \operatorname{lva}(G) \leq 3$. In order to show that $\operatorname{lva}(G)>2$, assume that the vertex set of $G$ is colored black and white where each monochromatic component induces a linear forest. Without loss of generality, we may assume that the central vertex is white. Since the central vertex cannot have more than two white neighbors, at most two other vertices are white. Note that the neighbors of the central vertex form a square in Fig. 8 and that each side of the square contains a cycle. Hence, none of the sides of the square can be monochromatic; it must contain at least one white vertex. Therefore, the boundary of the square contains exactly two white vertices, which must be placed in opposite corners. If the white vertices are placed in the top left and the bottom right corners, then the two other corners, which are black, have three black neighbors. If the white vertices are placed in the top right and the bottom left corners, then the three white vertices induce a cycle. In both cases, we have a contradiction.

Remark 3.2. A computer verification shows that all planar graphs $G$ with up to 8 vertices have lva $(G) \leq 2$. Counting the number of edges, the smallest planar graph $G$ with lva $(G) \geq 3$ is depicted in Fig. 7.

\subsection{Placing Vertices on Few Lines in the Plane $\left(\pi_{2}^{1}\right)$}

We start showing lower bounds for the parameter $\pi_{2}^{1}(G)$. Recall that the dual of a 3connected planar graph $G$ is a graph $G^{*}$ whose vertices are the faces of $G$ (represented by their facial cycles). The definition does not depend on a particular embedding of $G$ in the plane by Whitney's theorem, which says that all embeddings of a 3-connected planar graph in the sphere are equivalent up to a homeomorphism (therefore, the set of facial cycles of $G$ does not depend on a particular plane embedding). Two faces are adjacent in $G^{*}$ if and only if they share a common edge. The dual graph $G^{*}$ is also a 3-connected planar graph, and $\left(G^{*}\right)^{*}$ is isomorphic to $G$. Note that the dual of any cubic 3-connected planar graph is a triangulation. Conversely, the dual of a triangulation is a cubic graph. 
Recall that the circumference of a graph $G$, denoted by $c(G)$, is the length of a longest cycle in $G$. For a planar graph $G$, let $\bar{v}(G)$ denote the maximum $k$ such that $G$ has a straight-line plane drawing with $k$ collinear vertices.

Lemma 3.3. For any planar graph $G$ with $n$ vertices, the following holds:

(a) $\pi_{2}^{1}(G) \geq n / \bar{v}(G)$.

(b) If $G$ is a triangulation, then $\pi_{2}^{1}(G) \geq(2 n-4) / c\left(G^{*}\right)$.

Proof. Since part (a) is obvious, we only prove part (b). Let $\gamma(G)$ denote the minimum number of cycles in the dual graph $G^{*}$ sharing a common vertex and covering every vertex of $G^{*}$ at least twice. Note that, as $G$ is a triangulation, $\gamma(G) \geq(4 n-8) / c\left(G^{*}\right)$, where $2 n-4$ is the number of vertices in $G^{*}$ (as a consequence of Euler's formula). We now show $\pi_{2}^{1}(G) \geq \gamma(G) / 2$, which implies the claimed result.

Given a drawing realizing $\pi_{2}^{1}(G)$ with line set $\mathcal{L}$, for every line $\ell \in \mathcal{L}$, draw two parallel lines $\ell^{\prime}, \ell^{\prime \prime}$ sufficiently close to $\ell$ such that they together intersect the interiors of all faces touched by $\ell$ and do not go through any vertex of the drawing. Note that $\ell^{\prime}$ and $\ell^{\prime \prime}$ cross boundaries of faces only via inner points of edges. Each such crossing corresponds to a transition from one vertex to another along an edge in the dual graph $G^{*}$. Since all inner faces of $G$ are triangles, each of them is visited by each of $\ell^{\prime}$ and $\ell^{\prime \prime}$ at most once. Therefore, the faces crossed along $\ell^{\prime}$ and the faces crossed along $\ell^{\prime \prime}$, among them the outer face of $G$, each form a cycle in $G^{*}$. It remains to note that every face $f$ of the graph $G$ is crossed at least twice, because the boundary of $f$ is intersected by at least two different lines from $\mathcal{L}$ and each of these two lines has a parallel copy that crosses $f$.

An infinite family of triangulations $G$ with $\bar{v}(G) \leq n^{0.99}$ was constructed by Ravsky and Verbitsky [72]. By Lemma 3.3(a) this implies that there are infinitely many triangulations $G$ with $\pi_{2}^{1}(G) \geq n^{0.01}$. Lemma 3.3(b) along with an estimate of Grünbaum and Walther [49] (that was used also by Ravsky and Verbitsky [72]) yields a stronger result.

Theorem 3.4. There are infinitely many triangulations $G$ with $\Delta(G) \leq 12$ and $\pi_{2}^{1}(G) \geq n^{0.01}$.

Proof. The shortness exponent $\sigma_{\mathcal{G}}$ of a class $\mathcal{G}$ of graphs describes the length of the longest cycle in relation to the number of vertices for the graphs in the class. Formally, $\sigma_{\mathcal{G}}$ is the infimum of the set of the reals $\lim \inf _{i \rightarrow \infty} \log c\left(H_{i}\right) / \log \left|V\left(H_{i}\right)\right|$ for all sequences of $H_{i} \in \mathcal{G}$ such that $\left|V\left(H_{i}\right)\right|<\left|V\left(H_{i+1}\right)\right|$ [49]. Thus, for each $\epsilon>0$, there are infinitely many graphs $H \in \mathcal{G}$ with $c(H)<|V(H)|^{\sigma_{\mathcal{G}}+\epsilon}$. The dual graphs of triangulations with maximum vertex degree at most 12 are exactly the cubic 3-connected planar graphs with each face incident to at most 12 edges (this parameter is well defined by Whitney's theorem). Let $\sigma$ denote the shortness exponent for this class of graphs. It is known [49, Theorem 7] that $\sigma \leq \frac{\log 26}{\log 27}<0.989$. The theorem follows from this bound by Lemma 3.3(b).

This lower bound proves that we cannot get a constant upper bound for the weak line cover number in 2D. A similar result of Firman et al. [43] says that there is an infinite family of planar graphs with maximum degree 6, treewidth 3, and unbounded (logarithmic) $\pi_{2}^{1}$-value. In subsequent work, Eppstein [40] showed that for infinitely many values of $n$, 

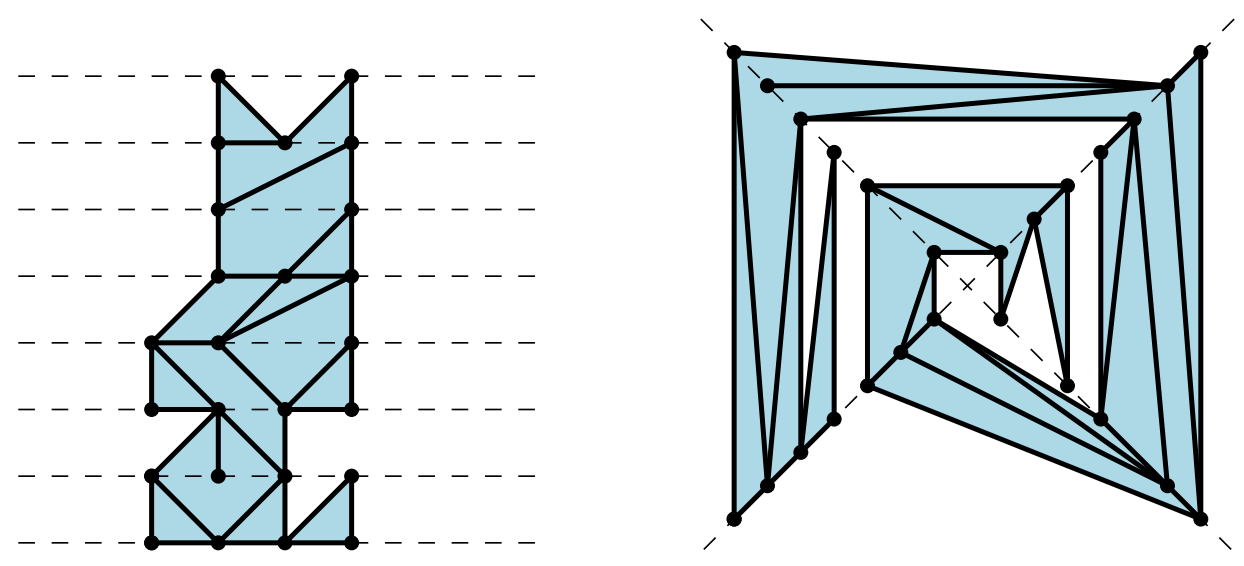

Figure 9. For the proof of Theorem 3.5.

there is a cubic, 3-connected, bipartite planar graph $G$ with $n$ vertices and $\pi_{2}^{1}(G) \in \Omega(\sqrt[3]{n})$. All three results, however, leave a gap to the trivial $O(n)$ upper bound for $\pi_{2}^{1}$. So we ask the following question.

Problem 4. Does $\pi_{2}^{1}(G)=o(n)$ hold for every (constant-degree) planar graph $G$ ?

Similarly to the weak line cover numbers in 3D, we can show constant upper bounds for some subclasses of planar graphs. A graph is weakly leveled planar ${ }^{3}$ [3] if it has a plane drawing in which the vertices are placed on a sequence of parallel lines, called tracks, such that every edge either lies on one of the tracks or its endpoints lie on two consecutive tracks. Obviously, the minimum number of tracks needed for a weakly leveled planar drawing of $G$ is an upper bound for $\bar{\pi}_{2}^{1}(G)$ (and thus also for $\pi_{2}^{1}(G)$ ) for any weakly leveled planar graph $G$.

The following theorem is similar to a lemma of Bannister et al. [3, Lemma 2] who say it is implicit in the earlier work of Felsner et al. [42].

Theorem 3.5. Any weakly leveled planar graph $G$ satisfies $\pi_{2}^{1}(G) \leq 2$.

Proof. We consider a weakly leveled plane drawing of $G$ and show how to transform it into a drawing on two intersecting lines; see Fig. 9. Put the tracks consecutively along a spiral so that they correspond to disjoint intervals on the half-lines as depicted on the right. Tracks whose indices are equal modulo 4 are placed on the same half-line. (Bannister et al. [3, Fig. 2] use three half-lines meeting in a point.)

Observe that any tree is weakly leveled planar: two vertices are aligned on the same track if and only if they are at the same distance from an arbitrarily assigned root. Moreover, any outerplanar graph is weakly leveled planar [42, Lemma 5]. This yields an improvement over the bound $\pi_{3}^{1}(G) \leq 2$ for outerplanar graphs stated in the previous section.

Corollary 3.6. Any outerplanar graph $G$ satisfies $\pi_{2}^{1}(G) \leq 2$.

\footnotetext{
${ }^{3}$ Felsner et al. [42] call this property track drawable; Suderman [80] calls it short layered drawable.
} 
Table 2. Summary of our results regarding the gaps between various graph parameters for certain planar graph classes.

\begin{tabular}{|c|c|c|c|c|c|c|}
\hline graph $G$ & $\operatorname{slope}(G)$ & $\leq$ & $\rho_{2}^{1}(G)$ & $\leq$ & $\operatorname{seg}(G)$ & Reference \\
\hline $\begin{array}{l}T_{k}=C_{3} \times P_{k} \\
m K_{2}\end{array}$ & $O(1)$ & & $\begin{array}{c}\Omega(n) \\
1\end{array}$ & & $m$ & Theorem 3.13 \\
\hline caterpillar of degree 3 & 2 & & $\Theta(\sqrt{n})$ & & $\Omega(n)$ & Example 3.7 \\
\hline certain triangulations & & & $O(\sqrt{n})$ & & $\Omega(n)$ & Example 3.8 \\
\hline any connected planar graph & & & & & $O\left(\left(\rho_{2}^{1}(G)\right)^{2}\right)$ & Theorem 3.11(a) \\
\hline graph $G$ & $\operatorname{la}(G)$ & $\leq$ & $\rho_{3}^{1}(G)$ & $\leq$ & $\rho_{2}^{1}(G)$ & Reference \\
\hline cubic graph & 2 & & $\Omega(\sqrt{n})$ & & & Remark 2.8 \\
\hline$T_{k}=C_{3} \times P_{k}$ & & & $O\left(n^{2 / 3}\right)$ & & $\Omega(n)$ & Theorem 3.13 \\
\hline
\end{tabular}

As is well known, the treewidth of an outerplanar graph is at most 2, and all graphs of treewidth 2 are planar but not all of them are weakly leveled planar. So one may wonder whether there is a constant $c \geq 2$ such that $\pi_{2}^{1}(G) \leq c$ for every graph $G$ of treewidth 2 . In subsequent work, however, Eppstein [40] constructed, for any $k>0$, a series-parallel graph $G$ with $\pi_{2}^{1}(G)>k$ and an apex-tree $T$ with $\pi_{2}^{1}(T)>k$. Both results show that there are graphs of treewidth 2 whose vertices cannot be covered by a constant number of lines.

\subsection{Placing Edges on Few Lines $\left(\rho_{2}^{1}\right.$ and $\left.\rho_{3}^{1}\right)$}

The parameter $\rho_{2}^{1}(G)$ of a graph $G$ is related to the parameters segment number $\operatorname{seg}(G)$ and slope number slope $(G)$ introduced by Dujmović et al. [29]. Clearly, we have the relation slope $(G) \leq \rho_{2}^{1}(G) \leq \operatorname{seg}(G)$, but these parameters can be far away from each other. Our results are summarized in Table 2. Figure 10 shows an example of a graph sequence with slope $(G)=O(1)$ and $\rho_{2}^{1}(G)=\Omega(n)$ (see the proof of Theorem 3.13 below). On the other hand, note that $\rho_{2}^{1}\left(m K_{2}\right)=1$ while $\operatorname{seg}\left(m K_{2}\right)=m$, where $m K_{2}$ denotes the graph consisting of $m$ isolated edges. The gap between $\rho_{2}^{1}(G)$ and $\operatorname{seg}(G)$ can be large even for connected graphs.

Example 3.7. For any $k$, let $G_{k}$ be the caterpillar with $k$ vertices of degree 3 and $k+2$ leaves. We have $\operatorname{seg}\left(G_{k}\right) \geq n / 2, \rho_{2}^{1}\left(G_{k}\right)=\Theta(\sqrt{n})$, and $\operatorname{slope}\left(G_{k}\right)=2$ where $n=2 k+2$ is the number of vertices of $G_{k}$.

Proof. It is not hard to see that, for any graph $G, \operatorname{seg}(G)$ is bounded from below by half the number of odd-degree vertices (see [29] for details). Therefore, $\operatorname{seg}\left(G_{k}\right) \geq n / 2$, whereas $\rho_{2}^{1}\left(G_{k}\right)=O(\sqrt{n})$. This is due to the fact that $G_{k}$ has a straight-line orthogonal drawing in a square grid of area $O(n)$. The lower bound $\rho_{2}^{1}\left(G_{k}\right)>\sqrt{n-2}$ follows by Lemma 2.7(a). Finally, slope $\left(G_{k}\right)=2$ as $G_{k}$ is a binary tree, which can be drawn on orthogonal lines. 


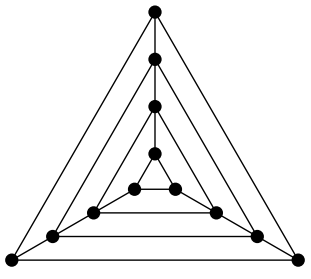

Figure 10. The nested-triangles graph $T_{k}=C_{3} \times P_{k}$ (here for $k=4$ ).

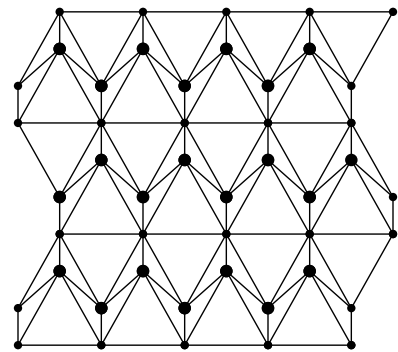

Figure 11. The main body of a triangulation $G$ with $\rho_{2}^{1}(G)=O(\sqrt{n})$ and $\operatorname{seg}(G)=\Omega(n)$.

It turns out that a large gap between $\rho_{2}^{1}(G)$ and $\operatorname{seg}(G)$ can also be shown for 3connected planar graphs and even for triangulations. Note that the following gap is the best possible because any 3 -connected graph $G$ has minimum vertex degree at least 3 and, hence, $\rho_{2}^{1}(G) \geq \rho_{3}^{1}(G)>\sqrt{2 n}$ by Lemma $2.7(\mathrm{a})$.

Example 3.8. There are triangulations with $\rho_{2}^{1}(G)=O(\sqrt{n})$ and $\operatorname{seg}(G)=\Omega(n){ }^{4}$

Proof. Consider the graph shown in Fig. 11. Its vertices are placed on the standard orthogonal grid and two slanted grids, which implies that at most $O(\sqrt{n})$ lines are involved. The pattern can be completed to a triangulation by adding three vertices around it and connecting them to the vertices on the pattern boundary. Since the pattern boundary contains $O(\sqrt{n})$ vertices, $O(\sqrt{n})$ new lines suffice for this. Thus, we have $\rho_{2}^{1}(G)=O(\sqrt{n})$ for the resulting triangulation $G$. Note that the vertices drawn fat in Fig. 11 have degree 5 , and there are linearly many of them. This implies that $\operatorname{seg}(G)=\Omega(n)$.

Somewhat surprisingly, $\operatorname{seg}(G)=O\left(\rho_{2}^{1}(G)^{2}\right)$ and even $\operatorname{seg}(G)=O\left(\rho_{3}^{1}(G)^{2}\right)$ for every connected graph $G$. The first bound follows from the following observation ${ }^{5}$, but for the second we need a more subtle analysis of the structure of $G$.

Lemma 3.9. Every drawing of a connected graph covered by $r \geq 2$ lines consists of at most $r(r-1)$ segments.

Proof. As the graph is connected, each segment in the drawing is intersected by at least one other segment. Thus each segment contains a crossing point of two lines covering the drawing. It follows that each of the $r$ straight lines can contain at most $r-1$ segments (one for each crossing) and, therefore, the drawing contains at most $r(r-1)$ segments.

It is easy to determine the segment number of graphs with maximum degree 2. For all other graphs we show the following upper bound on their segment number.

\footnotetext{
${ }^{4}$ A triangulation $G$ with $\operatorname{seg}(G)=O(\sqrt{n})$ has been found by Dujmović at al. [29, Fig. 12].

${ }^{5}$ Though we need Lemma 3.9 for 2-dimensional drawings, note that it holds true with literally the same proof also in the 3 -dimensional case.
} 
Lemma 3.10. For any connected planar graph $G$ with $\Delta(G) \geq 3$, we have

$$
\operatorname{seg}(G) \leq \sum_{\substack{v \in V(G) \\ \operatorname{deg} v \geq 3}} \operatorname{deg} v
$$

Proof. Call a vertex $v$ of the graph $G$ branching if its degree is at least three. A path between vertices of the graph $G$ will be called straight if it has no branching vertices other than its endpoints.

Reduce the graph $G$ to a graph $G^{\prime}$ as follows. The set of vertices of $G^{\prime}$ is the set of branching vertices in $G$, and two vertices are adjacent in $G^{\prime}$ if they are connected by a straight path in $G$. Being a planar graph, $G^{\prime}$ has a straight-line drawing.

Since $G$ is connected, every vertex in it is connected to a branching vertex by a straight path (possibly of zero length). Thus, we can construct a straight-line drawing of $G$ from a straight-line drawing of $G^{\prime}$ as follows. Note that an edge $e$ of $G^{\prime}$ corresponds to a bond of paths in $G$ connecting the incident vertices of $e$. We draw one path in the bond on the segment $e$, and each other is drawn as a pair of two segments that are close to $e$. A branching vertex $v$ in $G$ can be connected by a straight path to a degree-1 vertex (which disappears in $G^{\prime}$ ). We restore each such path by drawing it as a small segment. Moreover, $v$ can belong to a cycle whose all vertices except $v$ have degree 2 . We draw each such cycle as a small triangle.

Note that the segments incident to the branching vertex $v$ are split into three parts: $b_{v}$ segments which belong to the edge bonds, $l_{v}$ segments going to leaves, and $t_{v}$ segments that are sides of the small triangles. Unless $l_{v}=0$ and $t_{v}=2$, we can ensure that the last $l_{v}+t_{v}$ segments are drawn in at most $\left\lceil\left(l_{v}+t_{v}\right) / 2\right\rceil$ lines all crossing at the point $v$. Therefore, the vertex $v$ is incident to at most $b_{v}+\left\lceil\left(l_{v}+t_{v}\right) / 2\right\rceil$ segments. This holds true even if $l_{v}=0$ and $t_{v}=2$; we need to use the fact that in this case $b_{v} \neq 0$ since $\operatorname{deg} v \geq 3$. We will say that these segments are related to $v$. We also relate to $v$ the opposite sides of the corresponding small triangles; there are $t_{v} / 2$ of them. Thus, the vertex $v$ has at most

$$
b_{v}+\left\lceil\frac{l_{v}+t_{v}}{2}\right\rceil+\frac{t_{v}}{2} \leq b_{v}+\left\lceil\frac{l_{v}+t_{v}}{2}\right\rceil+\left\lfloor\frac{l_{v}+t_{v}}{2}\right\rfloor=b_{v}+l_{v}+t_{v}=\operatorname{deg} v
$$

segments. Since every segment of the constructed drawing of $G$ is related to some branching vertex, the total number of segments is bounded by $\sum_{v \in V(G), \operatorname{deg} v \geq 3} \operatorname{deg} v$.

Theorem 3.11. For any connected planar graph $G$ with at least two vertices, we have

(a) $\operatorname{seg}(G) \leq \rho_{2}^{1}(G)^{2}$, and

(b) $\operatorname{seg}(G)<2 \rho_{3}^{1}(G)^{2}$.

Proof. (a) The relation is an immediate consequence of Lemma 3.9.

(b) Suppose first that $\Delta(G) \geq 3$. Then Lemma 3.10 implies that

$$
\operatorname{seg}(G) \leq \sum_{\substack{v \in V(G) \\ \operatorname{deg} v \geq 3}} \operatorname{deg} v \leq 2 \sum_{\substack{v \in V(G) \\ \operatorname{deg} v \geq 3}}\left\lceil\frac{\operatorname{deg} v}{2}\right\rceil\left(\left\lceil\frac{\operatorname{deg} v}{2}\right\rceil-1\right) \leq 2 \rho_{3}^{1}(G)\left(\rho_{3}^{1}(G)-1\right)
$$


where the last estimation follows from the first inequality in the proof of Lemma 2.7(b).

If $\Delta(G) \leq 2$, then $G$ is a path or a cycle. The theorem holds true because we have $\operatorname{seg}(G)=\rho_{3}^{1}(G)=1$ in the former case and $\operatorname{seg}(G)=\rho_{3}^{1}(G)=3$ in the latter case.

In the inequality in the proof above, we upper bound $\operatorname{deg} v$ by a term quadratic in $\operatorname{deg} v$. It might look as if this bound is not tight, but in fact there is a quadratic gap between $\operatorname{seg}(G)$ and $\rho_{3}^{1}(G)$. For example, the caterpillars $G_{k}$ mentioned in Example 3.7 have $\operatorname{seg}\left(G_{k}\right)=\Omega(n)$ and $\rho_{3}^{1}\left(G_{k}\right) \leq \rho_{2}^{1}\left(G_{k}\right)=O(\sqrt{n})$.

Remark 3.12. For general, not necessarily connected, planar graphs, Lemma 3.10 implies the relation

$$
\operatorname{seg}(G)<p(G)+3 c(G)+2 \rho_{3}^{1}(G)^{2}<p(G)+\frac{5}{2} \rho_{3}^{1}(G)^{2}
$$

where $p(G)$ and $c(G)$ denote the number of connected components of $G$ that are paths and cycles, respectively.

Note that $\operatorname{la}(G) \leq \rho_{3}^{1}(G) \leq \rho_{2}^{1}(G) \leq \operatorname{seg}(G) \leq m$ for any planar graph $G$. For all inequalities here except the second one, we already know that the gap between the respective pair of parameters can be very large (by Remark 2.8, by Example 3.8, and by considering the path graph $P_{n}$, for which $\operatorname{seg}\left(P_{n}\right)=1$ ). The following theorem shows a large gap also between the parameters $\rho_{3}^{1}(G)$ and $\rho_{2}^{1}(G)$, that is, some planar graphs can be drawn much more efficiently, with respect to the line cover number, in 3-space than in the plane.

Theorem 3.13. For each $k$, there is a planar graph $G$ on $n=3 k$ vertices with $\Delta(G) \leq 4$, $\operatorname{tw}(G) \leq 3, \rho_{2}^{1}(G)=\Omega(n)$, and $\rho_{3}^{1}(G)=O\left(n^{2 / 3}\right)$.

Proof. Consider the nested-triangles graph $T_{k}=C_{3} \times P_{k}$ shown in Fig. 10. The graph $T_{k}$ has $n=3 k$ vertices. To prove the statement, it suffices to establish the following bounds:

(i) $\rho_{2}^{1}\left(T_{k}\right) \geq n / 2$ and

(ii) $\rho_{3}^{1}\left(T_{k}\right)=O\left(n^{2 / 3}\right)$.

To see the linear lower bound (i), note that $T_{k}$ is 3-connected. Hence, Whitney's theorem implies that, in any plane drawing of $T_{k}$, there is a sequence of nested triangles of length at least $k / 2$. The sides of the triangles in this sequence must belong to pairwise different lines. Therefore, $\rho_{2}^{1}\left(T_{k}\right) \geq 3 k / 2=n / 2$.

For the sublinear upper bound (ii), consider first the graph $C_{4} \times P_{k}$. We build wireframe rectangular prisms that are stacks of $O(\sqrt[3]{n})$ cubes each. These prisms are put onto the base plane in an $O(\sqrt[3]{n}) \times O(\sqrt[3]{n})$ grid. They allow us to place most of the edges of $C_{4} \times P_{k}$ on the $O\left(n^{2 / 3}\right)$ lines of the $3 \mathrm{D}$ cubic grid of volume $O(n)$; see Fig. 12 . Next, we fix a path that traverses all cubes by passing through the prisms from top to bottom (or vice versa) and connecting neighboring prisms. We add $O\left(n^{2 / 3}\right)$ intermediate edges connecting the neighboring prisms according to this path. For this "bending" we need $O\left(n^{2 / 3}\right)$ additional lines. The same approach works for the graph $T_{k}=C_{3} \times P_{k}$. In addition to the standard $3 \mathrm{D}$ grid, here we need also its slanted, diagonal version (and, again, additional lines for bending in the cubic box of volume $O(n)$ ). The number of lines increases just by a constant factor. 
Problem 5. Recall that, by Theorem 2.9, graphs of bounded degree can have linearly large parameter $\rho_{3}^{1}(G)$. If a planar graph $G$ has bounded degree, is it true that $\rho_{3}^{1}(G)=o(n)$ ?

In Example 2.13(b) we showed that $\rho_{2}^{1}\left(K_{2, q}\right)=\lceil(3 m-2) / 4\rceil$ for any $q \geq 2$, where $m$ is the number of edges in the complete bipartite graph $K_{2, q}$. Motivated by this result, we ask:

Problem 6. What is the smallest $c$ such that $\rho_{2}^{1}(G) \leq(c+o(1)) m$ for any planar graph $G$ ? Example 2.13(b) shows that $c \geq 3 / 4$.

It has been known for some time that any binary tree has a straight-line orthogonal drawing on a grid of size $O(\sqrt{n \log \log n}) \times O(\sqrt{n \log \log n})$ [15,79]. Recently this area bound was improved by Chan [14] to $n \cdot 2^{O\left(\log ^{*} n\right)}$ by using the old algorithm as the base case of a new recursive algorithm. The width and height of the drawing can be balanced (by plugging the width function $W_{0}(n)=2^{O\left(\log ^{*} n\right)} n / \log n$ and the height function $H_{0}(n)=2^{O\left(\log ^{*} n\right)} \log n$ that follow from Chan's Theorem 11 into Chan's Lemma 10, using $A=\sqrt{n} \log n$ ). Hence, for any binary tree $T$, it holds that $\rho_{2}^{1}(T)=\sqrt{n} \cdot 2^{O\left(\log ^{*} n\right)}$. For the special case of complete binary trees, we give the following lower and upper bounds.

Example 3.14. If $G$ is the complete binary tree of height $h \geq 1$ consisting of $n=2^{h+1}-1$ vertices, then $\rho_{2}^{1}(G)>\sqrt{n-3}$. On the other hand, $\rho_{2}^{1}(G) \leq 2 \sqrt{2} \sqrt{n+1}-3$ if $h$ is even and $\rho_{2}^{1}(G) \leq 3 \sqrt{n+1}-3$ otherwise.

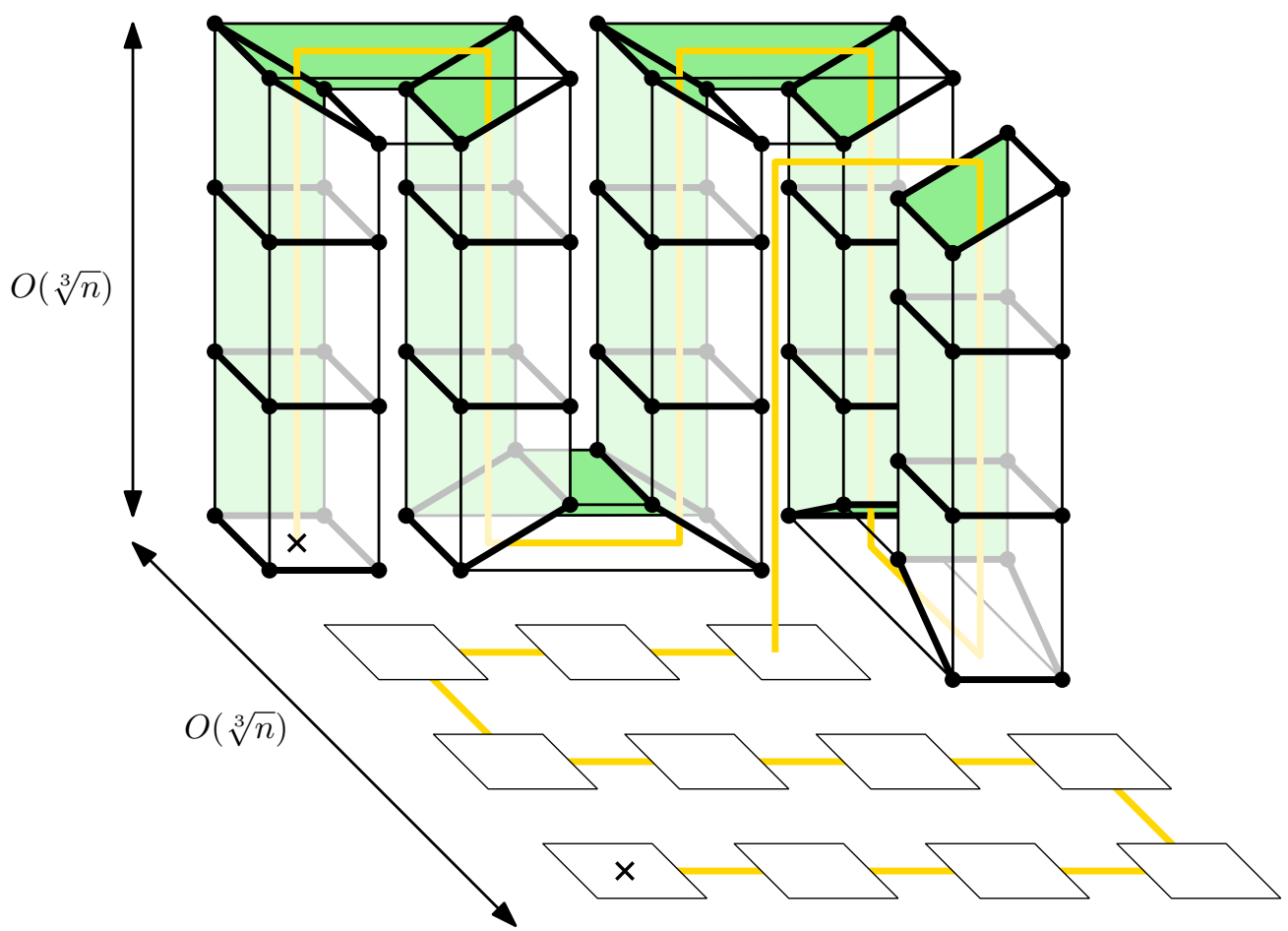

Figure 12. The graph $C_{4} \times P_{k}$ drawn into a 3D grid of linear volume on $O\left(n^{2 / 3}\right)$ lines (see the proof of Theorem 3.13). 


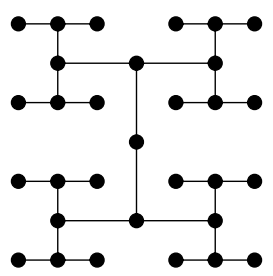

Figure 13. Binary tree of height 4 drawn using the approach by Shiloach [78, Fig. 31].

Proof. Indeed, since $G$ has $(n-3) / 2$ vertices of degree $3, \rho_{2}^{1}(G)>\sqrt{n-3}$ by Lemma 2.7 (a).

There are various ways to draw (complete) binary trees in an area-efficient way (e.g., by Crescenzi et al. [21, Section 4]). We use an approach presented by Shiloach in his thesis [78, page 95], providing the best known upper bounds. An example for a drawing using his layout is given in Figure 13. He draws the tree $G$ on a rectangular grid of size $W_{h} \times L_{h}$, where $W_{h}$ and $L_{h}$ are defined by the following formulas.

$$
W_{h}=\left\{\begin{array}{ll}
2^{\frac{h+2}{2}}-1 & \text { if } h \text { is even } \\
2^{\frac{h+1}{2}}-1 & \text { if } h \text { is odd }
\end{array} \quad L_{h}= \begin{cases}2^{\frac{h+2}{2}}-1 & \text { if } h \text { is even } \\
2^{\frac{h+3}{2}}-1 & \text { if } h \text { is odd }\end{cases}\right.
$$

Since $n=2^{h+1}-1$ and one of the grid lines through the root is redundant, we get the claimed upper bounds.

Complete binary trees and caterpillars of maximum degree 4 are the first non-trivial examples of bounded-degree trees. Both can be drawn on $O(\sqrt{n})$ lines. Can this be generalized to arbitrary binary trees?

Problem 7. Is it true that trees of bounded degree have a sublinear $\rho_{2}^{1}$-value? More specifically, is there a constant $c>0$ such that, for any tree $T, \rho_{2}^{1}(T) \leq c n^{1-1 / \Delta(T)}$ ? Even more specifically, is there a constant $c^{\prime}>0$ such that, for any binary tree $T, \rho_{2}^{1}(T) \leq c^{\prime} \sqrt{n}$ ?

It seems that, for weakly connected graphs such as trees, we cannot exploit the additional freedom that we have in $3 \mathrm{D}$ to get better bounds on the line cover number, so we conjecture that the following problem admits a positive answer.

Problem 8. Are $\rho_{3}^{1}(T)$ and $\rho_{2}^{1}(T)$ equal for every tree $T$ ?

\section{The Parallel Line Cover Numbers}

In this section we add the additional constraint that the lines that cover the drawing of the graph have to be parallel. First, we consider the parallel line cover number in 3-space and show its relation to the treewidth. We also state some relations to the affine cover numbers that we considered in the previous sections. Again, we show exact values for the parallel line cover numbers of complete (bipartite) graphs. Finally, we consider the parallel line cover number in the plane and show that there can be a linear gap compared to the (non-parallel) line cover number in the plane.

Note that the parallel plane cover number is discussed in Section 2.2 where we show in Theorem 2.4 that, for any graph $G, \bar{\pi}_{3}^{2}(G)=\pi_{3}^{2}(G)$. 


\subsection{Placing Vertices on Few Parallel Lines in 3-Space $\left(\bar{\pi}_{3}^{1}\right)$}

Although similar concepts had implicitly been studied in several earlier works [26, 42, 52], the concept of a proper track drawing was formally introduced by Dujmović et al. [33] in combinatorial terms with the following geometric meaning. We call a 3D drawing of a graph $G$ a proper track drawing if there are parallel lines, called tracks, such that every vertex of $G$ lies on one of the tracks and every edge connects vertices lying on two different tracks. Edges between the same two tracks are not allowed to cross each other. Furthermore, we call a drawing of $G$ an improper track drawing if we allow edges between consecutive vertices of the same track. The proper track number $\operatorname{tn}^{\mathrm{P}}(G)$ (improper track number $\operatorname{tn}^{\mathrm{i}}(G)$ ) of $G$ is the minimum number of tracks in a proper (improper) track drawing of $G$. Originally, track layouts have been studied in the context of minimizing the volume of 3D grid drawings where the following connection was discovered: An $n$-vertex graph has a $3 \mathrm{D}$ grid drawing of size $O(1) \times O(1) \times O(n)$ if and only if its proper track number is $O(1)[33,34]$. Note that the definition of track drawings is different from the concept of weakly leveled planar drawings (also called track drawings by some authors), which were introduced on page 23. While the definition above does not exclude crossings of two edges if they are between disjoint pairs of tracks, note that all such crossings can be removed by slightly shifting the vertices within each track. We, therefore, have

$$
\bar{\pi}_{3}^{1}(G)=\operatorname{tn}^{\mathrm{i}}(G)
$$

for any graph $G$. A simple argument [33, Lemma 2.2] shows the following relationship between the proper and improper track number of any graph $G$ :

$$
\operatorname{tn}^{\mathrm{p}}(G) / 2 \leq \operatorname{tn}^{\mathrm{i}}(G) \leq \operatorname{tn}^{\mathrm{p}}(G) .
$$

Therefore, the upper bounds for $\operatorname{tn}^{\mathrm{p}}(G)$ surveyed by Dujmović et al. [34, Table 1] imply also upper bounds on $\bar{\pi}_{3}^{1}(G)$ for different classes of graphs $G$. In particular, Dujmović at al. [33] proved that

$$
\operatorname{tn}^{\mathrm{p}}(G) \leq 3^{\mathrm{tw}(G)} \cdot 6^{\left(4^{\mathrm{tw}(G)}-3 \operatorname{tw}(G)-1\right) / 9}
$$

for any graph $G$. Note that any upper bound for $\bar{\pi}_{3}^{1}(G)$ implies also an upper bound for $\rho_{3}^{2}(G)$.

Lemma 4.1. For any graph $G, \rho_{3}^{2}(G) \leq\left(\begin{array}{c}\bar{\pi}_{3}^{1}(G) \\ 2\end{array}\right)$.

Proof. Any two parallel lines of the drawing lie in a plane, and all the edges are located in these planes.

Since $\bar{\pi}_{3}^{1}(G) \leq \operatorname{tn}^{\mathrm{P}}(G)$, Lemma 4.1 implies that the parameter $\rho_{3}^{2}(G)$ is bounded from above by a function of the treewidth of $G$.

Whether or not $\operatorname{tn}^{\mathrm{p}}(G)$ and, hence, $\bar{\pi}_{3}^{1}(G)$ is bounded for the class of planar graphs was a long-standing open problem [33]; it was also mentioned by Dujmović and Whitesides [36, Open Problem 14.2]. It was finally solved by Dujmović et al. [31, Theorem 53].

Later Pupyrev [70, Theorem 2] improved their bound considerably, showing that $\operatorname{tn}^{\mathrm{P}}(G) \leq 225$ for any planar graph $G$. The best known lower bounds are $\bar{\pi}_{3}^{1}(G)=\operatorname{tn}^{\mathrm{i}}(G) \geq 7$ 
for a planar graph $G$ [34, Cor. 10] and $\operatorname{tn}^{\mathrm{p}}(T) \geq 8$ for a planar 3-tree $T$ [70, Corollary 1]. On the other hand, for any planar 3-tree $T, \operatorname{tn}^{\mathrm{p}}(T) \leq 25$ [70, Theorem 1]. Pupyrev also improved and tightened bounds on the proper track number of several families of planar graphs. Among others, he gave lower-bound constructions of outerplanar and weakly leveled planar graphs matching existing upper bounds (of 5 and 6 , respectively).

Wood [85, Theorem 2] proved that for all $\Delta \geq 3$ and for all sufficiently large $n$ (depending on $\Delta$ ), there is a $\Delta$-regular $n$-vertex graph with $\operatorname{tn}^{\mathrm{p}}(G) \geq c \sqrt{\Delta} n^{1 / 2-1 / \Delta}$ for some absolute constant $c$, which implies that $\bar{\pi}_{3}^{1}(G)$ is unbounded even for graphs of bounded degree. On the other hand, Dujmović and Wood [37] showed that every graph with bounded maximum degree has a 3-dimensional grid drawing with volume $O\left(n^{3 / 2}\right)$, and the same bound holds for graphs from a proper minor-closed class. The latter bound was recently improved to $O(n)$ by Dujmović et al. [31, Theorem 40].

Theorem 4.2. Let $G$ be a graph. The following statements hold.

(a) If $\bar{\pi}_{3}^{1}(G) \leq 3$, then $G$ is planar and $\pi_{2}^{1}(G) \leq \bar{\pi}_{3}^{1}(G)$.

(b) If $G$ is planar and $\pi_{2}^{1}(G) \leq 2$, then $\bar{\pi}_{3}^{1}(G) \leq 4$.

(c) If $G$ is, moreover, weakly leveled planar, then $\bar{\pi}_{3}^{1}(G) \leq 3$.

Proof. (a) The case of $\bar{\pi}_{3}^{1}(G)=2$ is obvious. Suppose that $\bar{\pi}_{3}^{1}(G)=3$. Since any two parallel lines in $\mathbb{R}^{3}$ define a plane, the graph $G$ can be drawn on the three rectangular faces of a triangular prism in $\mathbb{R}^{3}$, created by the three parallel tracks. Therefore, $G$ is planar. Now pick a point outside of the prism, but close to its triangular base. Project the drawing of $G$ onto a plane that does not intersect the prism and that is close and parallel to the other triangular face of the prism. This yields a $2 \mathrm{D}$ drawing of $G$ whose vertices lie on 3 lines.

(b) Assume that the graph $G$ is drawn on two lines $\ell_{1}$ and $\ell_{2}$. If these lines are parallel then $\bar{\pi}_{3}^{1}(G) \leq \bar{\pi}_{2}^{1}(G) \leq 2$ and we are done. If the lines $\ell_{1}$ and $\ell_{2}$ intersect in a point $O$, then the union $\ell_{1} \cup \ell_{2}$ is split into three open rays and one half-open ray. We can put the vertices from the rays into four parallel tracks, preserving their order from the point $O$ to infinity along the rays.

(c) Felsner et al. [42, Theorem 5] showed how any outerplanar graph can be drawn on a triangular prism by wrapping it around, which is an approach similiar to that in Theorem 3.5. They remarked that their construction can also be applied to weakly leveled planar graphs. The vertices of the drawing lie on the edges of the prism, which are the three parallel lines of our 3D drawing.

Example 4.3. The parallel line cover number of complete (bipartite) graphs is as follows.

(a) $\bar{\pi}_{3}^{1}\left(K_{n}\right)=n-1$ for any $n \geq 2$.

(b) $\bar{\pi}_{3}^{1}\left(K_{p, q}\right)=p+1$ for any $p \leq q$ and $q \geq 3$.

Proof. (a) Suppose that $K_{n}$ is drawn in $\mathbb{R}^{3}$. Obviously, no line in $\mathbb{R}^{3}$ contains more than two vertices of the drawing. Moreover, there are no two parallel lines containing two vertices each as this would imply that a pair of edges cross. We conclude that any set of 
parallel lines has at most one line containing more than one vertex, which implies the lower bound $\bar{\pi}_{3}^{1}\left(K_{n}\right) \geq n-1$.

The upper bound $\bar{\pi}_{3}^{1}\left(K_{n}\right) \leq n-1$ is provided by an arbitrary $3 \mathrm{D}$ drawing of the graph $K_{n}$. Indeed, we can cover two arbitrary vertices by a line $\ell$, and then $n-2$ lines parallel to $\ell$ are enough to cover the remaining $n-2$ vertices.

(b) (for the proper track number, the case $p=q$ was also considered by Dujmovic and Whitesides [36]). To show the upper bound $\bar{\pi}_{3}^{1}\left(K_{p, q}\right) \leq p+1$, we put the independent set of $q$ vertices on a straight line and use a separate straight line for each of the other $p$ vertices. To show the lower bound, let $\mathcal{L}$ be an optimal set of straight lines. We assume that our bipartition is defined by $p$ white and $q$ black vertices. First, suppose that one straight line $\ell \in \mathcal{L}$ contains a pair of vertices of the same color. Since our straight lines are parallel, no other straight line may contain two vertices of the other color as this would produce a crossing of the edges in between. Clearly, if $\ell$ is monochromatic, there are at least $p+1$ straight lines. If $\ell$ is not monochromatic, then $\ell$ contains exactly three vertices, where the monochromatic pair is separated by the other vertex. However, now, every line $\ell^{\prime} \in \mathcal{L} \backslash\{\ell\}$ must be monochromatic, otherwise the edges spanning between $\ell$ and $\ell^{\prime}$ will produce a crossing. Since $q \geq 3$, the total number of straight lines is at least $1+p-1+q-2 \geq p+1$.

In the other case, no line contains two monochromatic vertices. If $p<q$, then we already have our lower bound of $p+1$. However, if $p=q$, we need to argue a little more carefully. Here, we note that there cannot be three lines with two vertices each since this would imply a crossing. Note that, in order to avoid a crossing between the pair of edges connecting two lines, the order of the colors on the first line must be reversed on the second. In particular, with three lines, some pair of lines will violate this condition. Thus the total number of lines is at least $2+q-2+p-2 \geq p+1$, because $q \geq 3$.

\subsection{Placing Vertices on Few Parallel Lines in the Plane $\left(\bar{\pi}_{2}^{1}\right)$}

In this subsection, we consider only planar graphs.

If $\Delta(G) \leq 2$, then $G$ is a union of cycles and paths and, hence, $\pi_{2}^{1}(G)=\bar{\pi}_{2}^{1}(G) \leq 2$. When we relax the degree restriction to $\Delta(G) \leq 3$, the parameters $\pi_{2}^{1}(G)$ and $\bar{\pi}_{2}^{1}(G)$ can be different. As a simplest example, note that $\pi_{2}^{1}\left(K_{4}\right)=2$ while $\bar{\pi}_{2}^{1}\left(K_{4}\right)=3$. In general, the gap is unbounded. For example, for any tree $G$, we have $\pi_{2}^{1}(G) \leq 2$ by Theorem 3.5. On the other hand, Felsner et al. [42] showed that $\bar{\pi}_{2}^{1}(G) \geq \log _{3}(2 n+1)$ for every complete ternary tree $G$.

For graphs of maximum vertex degree 3 as well as for differently restricted graphs, we can show a much larger gap. We need the following notions. In a poly-line drawing of a graph the edges are represented by polygonal chains. The bends of an edge are the points where the edge changes its slope, that is, bends are points common to two consecutive segments of the polygonal chain. In a poly-line grid drawing both vertices and bends have integer coordinates. The height of a poly-line grid drawing is the height of its bounding box. Given a graph $G$, we denote by $\hat{h}(G)$ the smallest height of a poly-line grid drawing of $G$.

Lemma 4.4. For any non-empty planar graph $G, \bar{\pi}_{2}^{1}(G) \geq \hat{h}(G)$. 
Proof. To prove the lemma, it suffices to convert a given drawing of $G$ with vertices covered by horizontal lines $\ell_{1}, \ldots, \ell_{r}$ (ordered from bottom to top) to a poly-line grid drawing of $G$ on a grid of height $r$. Let $\hat{G}$ be a geometric graph obtained from $G$ by subdividing each edge $e$ in the drawing of $G$ at the intersection points of $e$ with the covering lines $\ell_{1}, \ldots, \ell_{r}$, that is, each edge is replaced by a path whose length depends on the number of covering lines intersected by the edge. For each $i \in\{1, \ldots, r\}$, let $v_{i, 1}, \ldots, v_{i, n(i)}$ be the vertices of $\hat{G}$ drawn on $\ell_{i}$ and ordered from left to right. Consider a straight-line drawing of $\hat{G}$ on the grid where a vertex $v_{i, j}$ is placed at the point $(i, j)$ of the plane for each pair $(i, j)$ with $1 \leq j \leq n(i)$. Since we keep the order of the vertices on each horizontal line, this drawing is crossing-free, so it induces a poly-line grid drawing of $G$.

A planar drawing of a graph has $k$ 1-fused stacked cycles $C_{1}, \ldots, C_{k}$ if the cycles are nested in this order and each pairwise intersection of two cycles consists of at most one vertex, that is, for $1 \leq i<k, C_{i}$ surrounds $C_{i+1}$ and $\left|C_{i} \cap C_{i+1}\right| \leq 1$. If the cycles are fully vertex-disjoint, we call them also just stacked cycles. See Fig. 14 for examples of 1-fused stacked cycles and stacked cycles.

The stacked cycle argument (see, for instance, [9, 23, 63]) allows Biedl [5,8] to show the following:

Lemma 4.5 (Biedl [5,8]). The bounding box of any planar poly-line drawing has width and height at least $2 k$ if it has $k$ stacked cycles, and it has width and height at least $k+1$ if it has $k$ 1-fused stacked cycles.

The lemma immediately leads to the following results on 3-connected planar graphs. Recall that a graph is called $k$-outerplanar if it has a planar drawing that becomes outerplanar when repeatedly removing the outer face $k-1$ times.

Theorem 4.6. For each $k$, there are 3 -connected planar graphs $G_{1}^{k}, G_{2}^{k}$, and $G_{3}^{k}$ on $n_{1}=$ $2 k+1, n_{2}=3 k$ and $n_{3}=6(k-1)$ vertices, respectively, such that $\pi_{2}^{1}\left(G_{1}^{k}\right), \pi_{2}^{1}\left(G_{2}^{k}\right), \pi_{2}^{1}\left(G_{3}^{k}\right) \leq 3$ and

1. $G_{1}^{k}$ is 2-outerplanar and $\bar{\pi}_{2}^{1}\left(G_{1}^{k}\right) \geq\left(n_{1}+3\right) / 4$,

2. $\Delta\left(G_{2}^{k}\right) \leq 4$ and $\bar{\pi}_{2}^{1}\left(G_{2}^{k}\right) \geq n_{2} / 3$,

3. $\Delta\left(G_{3}^{k}\right) \leq 3$ and $\bar{\pi}_{2}^{1}\left(G_{3}^{k}\right) \geq n_{3} / 6+1$.

Proof. Figure 14 depicts our choices for $G_{1}^{k}, G_{2}^{k}$ (the nested-triangles graph $T_{k}$ ), and $G_{3}^{k}$. The drawings certify that $\pi_{2}^{1}\left(G_{1}^{k}\right), \pi_{2}^{1}\left(G_{2}^{k}\right)$, and $\pi_{2}^{1}\left(G_{3}^{k}\right)$ are all bounded from above by 3 . (Note that the bent edges in the drawing of $G_{3}^{k}$ can easily be straightened.)

Biedl $[5,8]$ observed the following. The depicted drawings consist of $k$ 1-fused stacked cycles in the case of $G_{1}^{k}$ and of $k$ stacked cycles in the case of $G_{2}^{k}$ and $G_{3}^{k}$. Since the graphs are 3-connected, Whitney's theorem implies that all planar drawings of each of the three graphs are homeomorphic up to the choice of the outer face. Thus, any planar drawing of $G_{1}^{k}$ consists of at least $k / 21$-fused stacked cycles and any planar drawing of $G_{2}^{k}$ or $G_{3}^{k}$ 


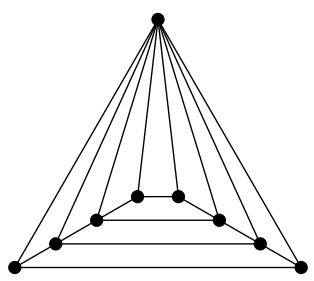

(a) $G_{1}^{k}$

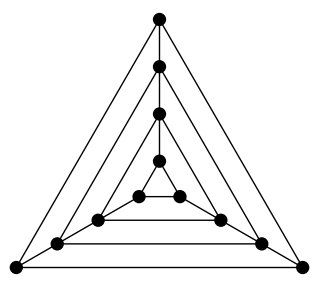

(b) $G_{2}^{k}$

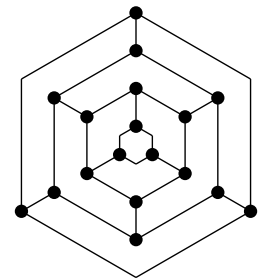

(c) $G_{3}^{k}$

Figure 14. Planar 3-connected graphs containing many (here $k=4$ ) nested cycles and therefore requiring $\Omega(k)$ parallel lines $[5,8]$.

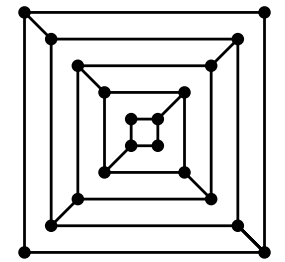

Figure 15. The nested-squares graph $S_{k}$ (here for $k=5$ ) [82].

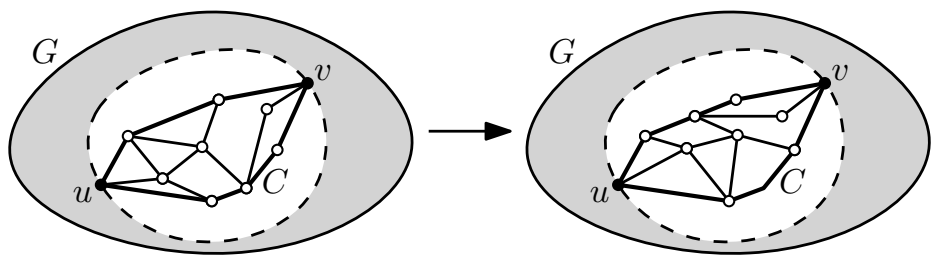

Figure 16. An example of a flipping operation.

consists of at least $k / 2$ stacked cycles. Consequently, by Lemma 4.5, we obtain

$$
\begin{aligned}
& \hat{h}\left(G_{1}^{k}\right) \geq k / 2+1=\left(n_{1}+3\right) / 4, \\
& \hat{h}\left(G_{2}^{k}\right) \geq \quad k \quad=n_{2} / 3, \text { and } \\
& \hat{h}\left(G_{3}^{k}\right) \geq \quad k \quad=n_{3} / 6+1 .
\end{aligned}
$$

Lemma 4.4 implies the theorem.

Now we turn our attention to 2-connected planar graphs.

Theorem 4.7. For each $k$, there is a planar graph $G$ on $n=4 k$ vertices with $\Delta(G) \leq 3$, $\pi_{2}^{1}(G)=2$, and $\bar{\pi}_{2}^{1}(G) \geq n / 4$.

Proof. Consider the graph $S_{k}$ (see also Valiant [82, Fig. 3]) consisting of $k=n / 4$ nested copies of $C_{4}$ connected as depicted in the drawing $H_{k}$ in Fig. 15.

Hence, this drawing consists of $k$ stacked cycles. Below we prove that any plane representation of $S_{k}$ consists of at least $k / 2$ stacked cycles. By Lemma $4.5, \hat{h}\left(S_{k}\right) \geq k$ and by Lemma $4.4, \bar{\pi}_{2}^{1}\left(S_{k}\right) \geq k=n / 4$. Figure 15 also certifies that $\pi_{2}^{1}\left(S_{k}\right)=2$.

Note that $S_{k}$ is 2-connected. We use general facts about plane embeddings of 2connected graphs; here we do not restrict ourselves to straight-line drawings only. A 2connected planar graph $G$ can have many plane representations, but all of them are obtainable from each other by a sequence of simple transformations. Specifically, let $H$ be a plane representation of $G$, and let $C$ be a cycle in $H$ containing only two vertices, $u$ and $v$, that are incident to some edges outside the region surrounded by $C$. We can obtain another plane embedding $H^{\prime}$ of $G$ by flipping $G$ with respect to $C$, that is, by replacing the interior of $C$ 
with its mirror version (up to homeomorphism); see Fig. 16. The rest of $G$ is unchanged; in particular, $u$ and $v$ keep their location. It turns out [64, Theorem 2.6.8] that, for any other plane representation $H_{1}$ of $G, H$ can be transformed into $H_{1}$ by a sequence of flippings that is followed, if needed, by re-assigning the outer face and applying a plane homeomorphism.

Let us apply this to $S_{k}$ and its plane representation $H_{k}$ of Fig. 15. First we need to identify all cycles in $H_{k}$ for which the flipping operation is possible. Recall that such a cycle $C$ is connected to its exterior only at two vertices $u$ and $v$. Clearly, the removal of these vertices disconnects the graph. This is possible only if $u$ and $v$ belong to two diagonal edges forming a centrally symmetric pair of edges. If the last condition is true for $u$ and $v$, then an appropriate cycle $C$ exists only if the pair $\{u, v\}$ is centrally symmetric. It readily follows from here that flipping is possible only with respect to square cycles except the outer one.

Note now that, for each such cycle $C$, the interior of $C$ is symmetric with respect to the axis passing through the corresponding vertices $u$ and $v$. This implies that flipping $H_{k}$ with respect to $C$ can be also seen just as a relabeling of vertices. Thus, the flipped graph has the same number of stacked cycles as before. Therefore, any further flipping also does not change the number of stacked cycles which remains to be $k$. Finally, by re-assigning the outer face and applying a plane homeomorphism, we obtain a plane representation of $S_{k}$ having at least $k / 2$ stacked cycles.

The following fact is straightforward (recall that a $2 \mathrm{D}$ grid drawing is straight-line).

Lemma 4.8. For any graph $G$, it holds that $\bar{\pi}_{2}^{1}(G) \leq h(G)$, where $h(G)$ denotes the minimum height of a $2 D$ grid drawing of $G$.

Using results by Biedl, we can apply this lemma to achieve results on series-parallel and outerplanar graphs.

\section{Corollary 4.9.}

(a) For any series-parallel graph $G$ with $n$ vertices, it holds that $\bar{\pi}_{2}^{1}(G)=O(\sqrt{n})$.

(b) For any outerplanar graph $G$ with $n$ vertices, it holds that $\pi_{2}^{1}(G)=O(\log n)$.

Proof. (a) Biedl [5, Theorem 1] showed that any series-parallel graph has a so-called flat visibility representation of area $O\left(n^{3 / 2}\right)$ and height $O(\sqrt{n})$. She [6] also showed that a flat visibility representation can be transformed into a straight-line grid drawing of the same height.

(b) Biedl [5, Corollary 2] proved that every outerplanar graph has a flat visibility representation of area $O(n \log n)$ without explicitly bounding the height. To bound the height and thus to be able to apply our Lemma 4.8 again, we need to look in her results in more detail. She defines the fan-out of a series-parallel graph as the maximum number of subgraphs used in a parallel composition. We augment the given graph $G$ to make it maximal outerplanar by adding additional edges. Those edges can be removed afterwards in the resulting straight-line drawing without increasing $\bar{\pi}_{2}^{1}(G)$. Biedl [5, Lemma 4.1] also proved that any maximal outerplanar graph is a series-parallel graph with fan-out at most 2 . 
According to Biedl [5, Theorem 2], her construction produces, given an $n$-vertex seriesparallel graph with fan-out $f$, a visibility representation of height $O(f \log n)$. This reduces to $O(\log n)$ in the case of outerplanar graphs. Although Biedl did not claim this explicitly, her representation is actually a flat visibility representation. (This fact is also mentioned in the proof of her Theorem 1). Again, this implies a straight-line grid drawing of the same height.

We remark that Frati [45] constructed a family of series-parallel graphs requiring a height of $\Omega\left(2^{\sqrt{\log n}}\right)$ in any poly-line grid drawing. By Lemma 4.4, these graphs have the same lower bound for $\bar{\pi}_{2}^{1}$.

We close this section by relating $\bar{\pi}_{2}^{1}$ to another well-known graph parameter; the pathwidth, which is defined similarly to treewidth (see, for example, [27]). For a given graph $G$, we denote its pathwidth by pw $(G)$. Based on [42, Theorem 1], Suderman [80, Lemma 25] notes that for every graph $G$, any unconstrained layered drawing of $G$ occupies at least $\mathrm{pw}(G)$ layers. In our notation, this means that $\mathrm{pw}(G) \leq \bar{\pi}_{2}^{1}(G)$. On the other hand, Dujmović et al. [33] showed that $\operatorname{tn}^{\mathrm{p}}(G) \leq \mathrm{pw}(G)$.

For trees, the pathwidth provides a constant-factor approximation of $\bar{\pi}_{2}^{1}$, because Suderman [80, Theorem 3] showed that $\bar{\pi}_{2}^{1}(T) \leq\lceil 3 \mathrm{pw}(T) / 2\rceil$ for any tree $T$. Other classes of planar graphs with bounded pathwidth, however, can have an unbounded $\bar{\pi}_{2}^{1}$-value. For instance, this is the case for the graphs of Theorem 4.6 (see Fig. 14) as their $\bar{\pi}_{2}^{1}$-values are in $\Omega(n)$ whereas their pathwidth is 3 [5, Theorems 4 and 5].

\section{Conclusion}

We have studied nine geometric graph parameters $-\rho_{2}^{1}, \rho_{3}^{1}, \rho_{3}^{2}, \pi_{2}^{1}, \pi_{3}^{1}, \pi_{3}^{2}, \bar{\pi}_{2}^{1}, \bar{\pi}_{3}^{1}, \bar{\pi}_{3}^{2}$. As it turned out, for any graph $G$, the following identities hold: $\pi_{3}^{1}(G)=\operatorname{lva}(G)$ (Theorem 2.1), $\pi_{3}^{2}(G)=\bar{\pi}_{3}^{2}(G)=\operatorname{vt}(G)$ (Theorem 2.4), $\bar{\pi}_{2}^{1}(G)$ is the minimum number of layers in an unconstrained layered drawing of $G$ (which Suderman [80] studied for trees), and $\bar{\pi}_{3}^{1}(G)=\operatorname{tn}^{\mathrm{i}}(G)$ (page 30), so only four of the parameters are new.

We have compared them among each other and with existing graph parameters. We have shown lower and upper bounds on some of the new parameters for certain graph classes such as trees, (outer)planar graphs, and complete (bipartite) graphs. In a companion paper [18] we have investigated, for some of the new parameters, the complexity of computing them for a given graph. In our study of the new parameters, we have identified eight open problems that could trigger further research in this area.

Acknowledgments. We thank a reviewer of an earlier version of this paper for finding and fixing an error in the proof of Theorem 2.14. We thank the reviewers of this version for the very detailed and valuable feedback, which helped to improve some of the results and to make the paper generally more accessible. 


\section{References}

[1] J. Akiyama, H. Era, S. V. Gervacio, and M. Watanabe. Path chromatic numbers of graphs. J. Graph Theory, 13(5):571-573, 1989. doi:10.1002/jgt.3190130506.

[2] J. Akiyama, G. Exoo, and F. Harary. Covering and packing in graphs III: Cyclic and acyclic invariants. Math. Slovaca, 30(4):405-417, 1980. URL: http://dml.cz/dmlcz/ 136252.

[3] M. J. Bannister, W. E. Devanny, V. Dujmović, D. Eppstein, and D. R. Wood. Track layouts, layered path decompositions, and leveled planarity. Algorithmica, 81(4):15611583, 2019. doi:10.1007/s00453-018-0487-5.

[4] J. Barát, J. Matoušek, and D. R. Wood. Bounded-degree graphs have arbitrarily large geometric thickness. Electr. J. Combin., 13:\#R3, 2006. doi:10.37236/1029.

[5] T. Biedl. Small drawings of outerplanar graphs, series-parallel graphs, and other planar graphs. Discrete Comput. Geom., 45(1):141-160, 2011. doi:10.1007/ s00454-010-9310-z.

[6] T. Biedl. Height-preserving transformations of planar graph drawings. In C. Duncan and A. Symvonis, editors, Proc. 22nd Int. Symp. Graph Drawing (GD'14), volume 8871 of Lect. Notes Comput. Sci., pages 380-391. Springer-Verlag, 2014. doi:10.1007/ 978-3-662-45803-7_32.

[7] T. Biedl, S. Felsner, H. Meijer, and A. Wolff. Line and plane cover numbers revisited. In D. Archambault and C. D. Tóth, editors, Proc. 27th Int. Symp. Graph Drawing Es Network Vis. (GD'19), volume 11904 of Lect. Notes Comput. Sci., pages 409-415. Springer-Verlag, 2019. arXiv:1908.07647. doi:10.1007/978-3-030-35802-0_31.

[8] T. C. Biedl. Small poly-line drawings of series-parallel graphs. Technical Report CS2007-23, School of Computer Science, University of Waterloo, Canada, 2005. URL: https://cs. uwaterloo.ca/research/tr/2007/CS-2007-23.pdf.

[9] T. C. Biedl and F.-J. Brandenburg. Drawing planar bipartite graphs with small area. In Proc. Canad. Conf. Comput. Geom. (CCCG'05), pages 105-108, 2005. URL: http: //www.cccg.ca/proceedings/2005/11.pdf.

[10] B. Bollobás. Combinatorics: Set Systems, Hypergraphs, Families of Vectors and Combinatorial Probability. Cambridge University Press, 1st edition, 1986.

[11] P. Bose, V. Dujmović, F. Hurtado, S. Langerman, P. Morin, and D. R. Wood. A polynomial bound for untangling geometric planar graphs. Discrete Comput. Geom., 42(4):570-585, 2008. doi:10.1007/s00454-008-9125-3.

[12] I. Broere and C. M. Mynhardt. Generalized colorings of outerplanar and planar graphs. In Y. Alavi, G. Chartrand, L. Lesniak, D. R. Lick, and C. E. Wall, editors, Proc. 5th Conf. Graph Theory with Applications to Algorithms and Computer Science, pages 151$161,1985$. 
[13] R. L. Brooks. On colouring the nodes of a network. Mathematical Proceedings of the Cambridge Philosophical Society, 37(2):194-197, 1941. doi:10.1017/ S030500410002168X.

[14] T. M. Chan. Tree drawings revisited. Discrete Comput. Geom., 63(4):799-820, 2020. doi:10.1007/s00454-019-00106-w.

[15] T. M. Chan, M. T. Goodrich, S. R. Kosaraju, and R. Tamassia. Optimizing area and aspect ratio in straight-line orthogonal tree drawings. Comput. Geom. Theory Appl., 23(2):153-162, 2002. doi:10.1016/S0925-7721(01)00066-9.

[16] L. S. Chandran and C. R. Subramanian. Girth and treewidth. J. Comb. Theory, Ser. B, 93(1):23-32, 2005. doi:10.1016/j.jctb.2004.05.004.

[17] S. Chaplick, K. Fleszar, F. Lipp, A. Ravsky, O. Verbitsky, and A. Wolff. Drawing graphs on few lines and few planes. In Y. Hu and M. Nöllenburg, editors, Proc. 24th Int. Symp. Graph Drawing \&6 Network Vis. (GD'16), volume 9801 of Lect. Notes Comput. Sci., pages 166-180. Springer-Verlag, 2016. arXiv:1607.01196. doi:10.1007/978-3-319-50106-2_14.

[18] S. Chaplick, K. Fleszar, F. Lipp, A. Ravsky, O. Verbitsky, and A. Wolff. The complexity of drawing graphs on few lines and few planes. In F. Ellen, A. Kolokolova, and J.R. Sack, editors, Proc. Algorithms Data Struct. Symp. (WADS'17), volume 10389 of Lect. Notes Comput. Sci., pages 265-276. Springer-Verlag, 2017. arXiv:1607.06444. doi:10.1007/978-3-319-62127-2_23.

[19] G. Chartrand and H. V. Kronk. The point-arboricity of planar graphs. J. Lond. Math. Soc., 44:612-616, 1969. doi:10.1112/jlms/s1-44.1.612.

[20] Z. Chen and X. He. Parallel complexity of partitioning a planar graph into vertex-induced forests. Discrete Appl. Math., 69(1-2):183-198, 1996. doi:10.1016/ 0166-218X (96) 00089-3.

[21] P. Crescenzi, G. Di Battista, and A. Piperno. A note on optimal area algorithms for upward drawings of binary trees. Computational Geometry, 2(4):187-200, 1992. doi:10.1016/0925-7721(92)90021-J.

[22] G. Da Lozzo, V. Dujmović, F. Frati, T. Mchedlidze, and V. Roselli. Drawing planar graphs with many collinear vertices. J. Comput. Geom., 9(1):94-130, 2018. doi: 10.20382/jocg.v9i1a4.

[23] H. de Fraysseix, J. Pach, and R. Pollack. Small sets supporting Fáry embeddings of planar graphs. In Proc. 20th Annu. ACM Symp. Theory Comput. (STOC'88), pages 426-433, 1988. doi:10.1145/62212.62254.

[24] H. de Fraysseix, J. Pach, and R. Pollack. How to draw a planar graph on a grid. Combinatorica, 10(1):41-51, 1990. doi:10.1007/BF02122694.

[25] G. Di Battista, F. Frati, and J. Pach. On the queue number of planar graphs. SIAM J. Comput., 42(6):2243-2285, 2013. doi:10.1137/130908051. 
[26] E. Di Giacomo, G. Liotta, and H. Meijer. Computing straight-line 3D grid drawings of graphs in linear volume. Computational Geometry, 32(1):26-58, 2005. doi:10.1016/ j.comgeo.2004.11.003.

[27] R. Diestel. Graph Theory. Springer-Verlag, Heidelberg, 5th edition, 2016.

[28] V. Dujmović. Graph layouts via layered separators. J. Comb. Theory, Ser. B, 110:7989, 2015. doi:10.1016/j.jctb.2014.07.005.

[29] V. Dujmović, D. Eppstein, M. Suderman, and D. R. Wood. Drawings of planar graphs with few slopes and segments. Comput. Geom. Theory Appl., 38(3):194-212, 2007. doi:10.1016/j.comgeo.2006.09.002.

[30] V. Dujmović, F. Frati, D. Gonçalves, P. Morin, and G. Rote. Every collinear set in a planar graph is free. In T. M. Chan, editor, Proc. 30th Ann. ACM-SIAM Symp. Discrete Algorithms (SODA'19), pages 1521-1538, 2019. arXiv:1811.03432. doi: 10.1137/1.9781611975482.92.

[31] V. Dujmović, G. Joret, P. Micek, P. Morin, T. Ueckerdt, and D. R. Wood. Planar graphs have bounded queue-number. J. ACM, 67(4:22), 2020. arXiv:1904.04791. doi:10.1145/3385731.

[32] V. Dujmović and P. Morin. Dual circumference and collinear sets. In G. Barequet and Y. Wang, editors, Proc. 35th Int. Symp. Comput. Geom. (SoCG'19), volume 129 of LIPIcs, pages 29:1-29:17. Schloss Dagstuhl - Leibniz-Zentrum für Informatik, 2019. doi:10.4230/LIPICs.SoCG.2019.29.

[33] V. Dujmović, P. Morin, and D. R. Wood. Layout of graphs with bounded tree-width. SIAM J. Comput., 34(3):553-579, 2005. doi:10.1137/S0097539702416141.

[34] V. Dujmović, A. Pór, and D. R. Wood. Track layouts of graphs. Disc. Math. Theor. Comput. Sci., 6(2):497-522, 2004. URL: https://dmtcs.episciences.org/315.

[35] V. Dujmović, M. Suderman, and D. R. Wood. Graph drawings with few slopes. Comput. Geom. Theory Appl., 38(3):181-193, 2007. doi:10.1016/j.comgeo.2006.08.002.

[36] V. Dujmović and S. Whitesides. Three-dimensional drawings. In R. Tamassia, editor, Handbook of Graph Drawing and Visualization, chapter 14, pages 455-488. CRC Press, 2013.

[37] V. Dujmović and D. R. Wood. Three-dimensional grid drawings with sub-quadratic volume. In J. Pach, editor, Towards a theory of geometric graphs, volume 342 of Contemporary Mathematics, pages 55-66. AMS, Providence, RI, 2004.

[38] S. Durocher and D. Mondal. Drawing plane triangulations with few segments. Computational Geometry, 77:27-39, 2019. doi:10.1016/j.comgeo.2018.02.003.

[39] S. Durocher, D. Mondal, R. Nishat, and S. Whitesides. A note on minimum-segment drawings of planar graphs. J. Graph Algorithms Appl., 17(3):301-328, 2013. doi: $10.7155 /$ jgaa. 00295 . 
[40] D. Eppstein. Cubic planar graphs that cannot be drawn on few lines. In G. Barequet and Y. Wang, editors, Proc. 35th Int. Symp. Comput. Geom. (SoCG'19), volume 129 of LIPIcs, pages 32:1-32:15. Schloss Dagstuhl - Leibniz-Zentrum für Informatik, 2019. doi:10.4230/LIPICs.SoCG. 2019.32.

[41] S. Felsner. 4-connected triangulations on few lines. In D. Archambault and C. D. Tóth, editors, Proc. 27th Int. Symp. Graph Drawing \& Network Vis. (GD'19), volume 11904 of Lect. Notes Comput. Sci., pages 395-408. Springer-Verlag, 2019. arXiv:1908.04524. doi:10.1007/978-3-030-35802-0_30.

[42] S. Felsner, G. Liotta, and S. Wismath. Straight-line drawings on restricted integer grids in two and three dimensions. J. Graph Algorithms Appl., 7(4):363-398, 2003. doi:10.7155/jgaa.00075.

[43] O. Firman, A. Ravsky, and A. Wolff. On the weak line cover numbers. In M. Korman and W. Mulzer, editors, Proc. 34th Europ. Workshop Comput. Geom. (EuroCG'18), pages 63:1-5, 2018. URL: https://conference.imp.fu-berlin.de/ eurocg18/download/paper_63.pdf.

[44] J. Flum and M. Grohe. Parametrized Complexity Theory. Springer-Verlag, Berlin, 2006.

[45] F. Frati. Lower bounds on the area requirements of series-parallel graphs. Disc. Math. Theor. Comput. Sci., 12(5), 2010. URL: https://dmtcs.episciences.org/500.

[46] X. Goaoc, J. Kratochvíl, Y. Okamoto, C.-S. Shin, A. Spillner, and A. Wolff. Untangling a planar graph. Discrete Comput. Geom., 42(4):542-569, 2009. doi:10.1007/ s00454-008-9130-6.

[47] W. Goddard. Acyclic colorings of planar graphs. Discrete Math., 91(1):91-94, 1991. doi:10.1016/0012-365X (91)90166-Y.

[48] R. L. Graham, D. E. Knuth, and O. Patashnik. Concrete Mathematics: A Foundation for Computer Science. Addison-Wesley Publishing Group, Amsterdam, 2nd edition, 1994.

[49] B. Grünbaum and H. Walther. Shortness exponents of families of graphs. J. Comb. Theory, Ser. A, 14(3):364-385, 1973. doi:10.1016/0097-3165(73)90012-5.

[50] H. Hanani. The existence and construction of balanced incomplete block designs. Ann. Math. Stat., 32(2):361-386, 1961. URL: http://www.jstor.org/stable/2237750.

[51] F. Harary. Covering and packing in graphs I. Ann. N.Y. Acad. Sci., 175(1):198-205, 1970. doi:10.1111/j.1749-6632.1970.tb56470.x.

[52] L. Heath and A. Rosenberg. Laying out graphs using queues. SIAM J. Comput., 21(5):927-958, 1992. doi:10.1137/0221055.

[53] U. Hoffmann. On the complexity of the planar slope number problem. J. Graph Algorithms Appl., 21(2):183-193, 2017. doi:10.7155/jgaa.00411. 
[54] G. Hültenschmidt, P. Kindermann, W. Meulemans, and A. Schulz. Drawing planar graphs with few geometric primitives. J. Graph Algorithms Appl., 22(2):357-387, 2018. doi:10.7155/jgaa.00473.

[55] A. Igamberdiev, W. Meulemans, and A. Schulz. Drawing planar cubic 3-connected graphs with few segments: Algorithms \& experiments. J. Graph Algorithms Appl., 21(4):561-588, 2017. doi:10.7155/jgaa.00430.

[56] R. E. Jamison. Few slopes without collinearity. Discrete Math., 60:199-206, 1986. doi: 10.1016/0012-365X(86)90012-9.

[57] V. Jelínek, E. Jelínková, J. Kratochvíl, B. Lidický, M. Tesař, and T. Vyskočil. The planar slope number of planar partial 3-trees of bounded degree. J. Graphs Combin., 29(4):981-1005, 2013. doi:10.1007/s00373-012-1157-z.

[58] P. Kindermann, W. Meulemans, and A. Schulz. Experimental analysis of the accessibility of drawings with few segments. J. Graph Algorithms Appl., 22(3):501-518, 2018. doi:10.7155/jgaa.00474.

[59] A. Kostochka and L. Melnikov. On a lower bound for the isoperimetric number of cubic graphs. In Proc. 3rd. Int. Petrozavodsk. Conf. Probabilistic Methods in Discrete Mathematics, pages 251-265. Moskva: TVP; Utrecht: VSP, 1993.

[60] M. Krein and D. Milman. On extreme points of regular convex sets. Studia Math., 9(1):133-138, 1940. URL: http://eudml.org/doc/219061.

[61] M. Kryven, A. Ravsky, and A. Wolff. Drawing graphs on few circles and few spheres. J. Graph Algorithms Appl., 23(2):371-391, 2019. doi:10.7155/jgaa.00495.

[62] M. Matsumoto. Bounds for the vertex linear arboricity. J. Graph Theory, 14(1):117126, 1990. doi:10.1002/jgt.3190140113.

[63] K. Miura, S.-I. Nakano, and T. Nishizeki. Grid drawings of 4-connected plane graphs. Discrete Comput. Geom., 26(1):73-87, 2001. doi:10.1007/s00454-001-0004-4.

[64] B. Mohar and C. Thomassen. Graphs on surfaces. John Hopkins University Press, Baltimore, MD, 2001.

[65] D. Mondal, R. I. Nishat, S. Biswas, and M. S. Rahman. Minimum-segment convex drawings of 3-connected cubic plane graphs. J. Comb. Optim., 25(3):460-480, 2013. doi:10.1007/s10878-011-9390-6.

[66] J. Pach and D. Pálvölgyi. Bounded-degree graphs can have arbitrarily large slope numbers. Electr. J. Combin., 13:\#N1, 4 pages, 2006. doi:10.37236/1139.

[67] J. Pach and G. Tardos. Untangling a polygon. Discrete Comput. Geom., 28(4):585-592, 2002. doi:10.1007/s00454-002-2889-y. 
[68] J. Pach, T. Thiele, and G. Tóth. Three-dimensional grid drawings of graphs. In G. Di Battista, editor, Proc. 5th Int. Symp. Graph Drawing (GD'97), volume 1353 of Lect. Notes Comput. Sci., pages 47-51. Springer-Verlag, 1997. doi:10.1007/ 3-540-63938-1_49.

[69] K. S. Poh. On the linear vertex-arboricity of a planar graph. J. Graph Theory, 14(1):7375, 1990. doi:10.1002/jgt.3190140108.

[70] S. Pupyrev. Improved bounds for track numbers of planar graphs. J. Graph Algorithms Appl., 24(3):323-341, 2020. doi:10.7155/jgaa.00536.

[71] A. Raspaud and W. Wang. On the vertex-arboricity of planar graphs. Eur. J. Comb., 29(4):1064-1075, 2008. doi:10.1016/j.ejc.2007.11.022.

[72] A. Ravsky and O. Verbitsky. On collinear sets in straight-line drawings. In P. Kolman and J. Kratochvíl, editors, Proc. 37th Int. Workshop Graph-Theoretic Concepts Comput. Sci. (WG'11), volume 6986 of Lect. Notes Comput. Sci., pages 295-306. Springer-Verlag, 2011. arXiv:0806.0253. doi:10.1007/978-3-642-25870-1_27.

[73] C. Reid and A. Rosa. Steiner systems $s(2,4, \nu)$ - a survey. Electr. J. Combin., DS18, 2010. doi:10.37236/39.

[74] M. Schaefer and D. Štefankovič. Fixed points, Nash equilibria, and the existential theory of the reals. Theory Comput. Syst., 60(2):172-193, 2017. doi:10.1007/ s00224-015-9662-0.

[75] U. Scherm. Minimale Überdeckung von Knoten und Kanten in Graphen durch Geraden. Bachelor's thesis, Institut für Informatik, Julius-Maximilians-Universität Würzburg, 2016. URL: http://www1.pub.informatik.uni-wuerzburg.de/pub/ theses/2016-scherm-bachelor .pdf.

[76] W. Schnyder. Embedding planar graphs on the grid. In D. S. Johnson, editor, Proc. 1st ACM-SIAM Symp. Discrete Algorithms (SODA'90), pages 138-148, 1990. URL: https://dl.acm.org/citation. cfm?id=320191.

[77] A. Schulz. Drawing graphs with few arcs. J. Graph Algorithms Appl., 19(1):393-412, 2015. doi:10.7155/jgaa.00366.

[78] Y. Shiloach. Linear and planar arrangements of graphs. PhD thesis, Weizmann Institute of Science, 1976. URL: https://weizmann-primo.hosted.exlibrisgroup. $\mathrm{com} / \mathrm{primo}$-explore/fulldisplay?docid=972WIS_ALMA1129639470003596\&context= L\&vid=972WIS_V1\&lang=en_US.

[79] C.-S. Shin, S. K. Kim, and K.-Y. Chwa. Area-efficient algorithms for straight-line tree drawings. Comput. Geom. Theory Appl., 15(4):175-202, 2000. doi:10.1016/ S0925-7721 (99) 00053-X.

[80] M. Suderman. Pathwidth and layered drawings of trees. Int. J. Comput. Geom. Appl., 14(3):203-225, 2004. doi:10.1142/S0218195904001433. 
[81] W. T. Tutte. How to draw a graph. Proc. London Math. Soc., 13:743-768, 1963. doi:10.1112/plms/s3-13.1.743.

[82] L. G. Valiant. Universality considerations in VLSI circuits. IEEE Trans. Comput., 30(2):135-140, 1981. doi:10.1109/TC.1981.6312176.

[83] J. Wang. On point-linear arboricity of planar graphs. Discrete Math., 72(1-3):381-384, 1988. doi:10.1016/0012-365X (88)90229-4.

[84] Wikipedia. Krein-Milman theorem. Accessed April 21, 2016. URL: https://en. wikipedia.org/wiki/Krein-Milman_theorem.

[85] D. R. Wood. Bounded-degree graphs have arbitrarily large queue-number. Disc. Math. Theor. Comput. Sci., 10(1):27-34, 2008. URL: http://dmtcs.episciences.org/434.

[86] D. R. Wood. Three-dimensional graph drawing. In M.-Y. Kao, editor, Encyclopedia of Algorithms, pages 1-7. Springer-Verlag, Boston, MA, 2008. doi:10.1007/ 978-3-642-27848-8_656-1. 